\title{
Enviromic assembly increases accuracy and reduces costs of the genomic prediction for yield plasticity
}

\author{
Germano Costa-Neto ${ }^{1 *}$, Jose Crossa ${ }^{2,3}$ and Roberto Fritsche-Neto ${ }^{1,4}$
}

${ }^{1}$ Department of Genetics, "Luiz de Queiroz" Agriculture College, University of São Paulo (ESALQ/USP),

5 Piracicaba, Brazil.

$6{ }^{2}$ Biometrics and Statistics Unit, International Maize and Wheat Improvement Center (CIMMYT), Carretera

México -Veracruz, Km 45, Col. El Batán, CP 56237, Texcoco, Edo. de México, México

${ }^{3}$ Colegio de Posgraduado, Montecillo, Texcoco, Edo. de México, México.

${ }^{4}$ Breeding Analytics and Data Management Unit, International Rice Research Institute (IRRI), Los Baños,

10 Philippines

\section{* Correspondence:}

12 Germano Costa-Neto

13 germano.cneto@gmail.com

14 Keywords: genomic selection, adaptability, genotype $\times$ environment, climate-smart; selective phenotyping

\section{ABSTRACT}

Quantitative genetics states that phenotypic variation is a consequence of genetic and environmental factors and their subsequent interaction. Here, we present an enviromic assembly approach, which includes the use of ecophysiology knowledge in shaping environmental relatedness into whole-genome predictions (GP) for plant breeding (referred to as E-GP). We propose that the quality of an environment is defined by the core of environmental typologies (envirotype) and their frequencies, which describe different zones of plant adaptation. From that, we derive markers of environmental similarity cost-effectively. Combined with the traditional genomic sources (e.g., additive and dominance effects), this approach may better represent the putative phenotypic variation across diverse growing conditions (i.e., phenotypic plasticity). Additionally, we couple a genetic algorithm scheme to design optimized multi-environment field trials (MET), combining enviromic assembly and genomic kinships to provide in-silico realizations of the future genotype-environment combinations that must be phenotyped in the field. As a proof-of-concept, we highlight E-GP applications: (1) managing the lack of phenotypic information in training accurate GP models across diverse environments and (2) guiding an early screening for yield plasticity using optimized phenotyping efforts. Our approach was tested using two non-conventional cross-validation schemes to better visualize the benefits of enviromic assembly in sparse experimental networks. Results on tropical maize show that E-GP outperforms benchmark GP in all scenarios and cases tested. We show that for training accurate GP models, the genotype-environment combinations' representativeness is more critical than the MET size. Furthermore, we discuss theoretical backgrounds underlying how the intrinsic envirotype-phenotype covariances within the phenotypic records of (MET) can impact the accuracy of GP and limits the potentialities of predictive breeding approaches. The E-GP is an efficient approach to better use environmental databases to deliver climate-smart solutions, reduce field costs, and anticipate future scenarios. 


\section{$40 \quad 1 \quad$ INTRODUCTION}

Environmental changing scenarios challenge agricultural research to deliver climate-smart solutions in a time-reduced and cost-effective manner (Tigchelaar et al., 2018; Ramírez-Villegas et al 2020; Cortés et al., 2020). Characterizing crop growth conditions is crucial for this purpose (Xu, 2016), allowing a deeper understanding of how the environment shapes past, present, and future phenotypic variations (e.g., Ramírez-Villegas et al. 2018; Heinemann et al., 2019; Cooper et al., 2014; de los Campos et al., 2020; Costa-Neto et al., 2021b; Antolin et al., 2021). For plant breeding research, mostly based on selecting the best-evaluated genotypes for a target population of environments (TPE), this approach is useful to discriminate genomic and non-genomic sources of crop adaptation. Thus, the concept of 'envirotyping' (environmental + typing, Cooper et al., 2014; Xu, 2016) emerges to establish

the quality of a given environment in the delivery of quality phenotypic records, mostly to train accurate

51 predictive breeding approaches capable of guiding the selection of most productive and adapted genotypes (Resende et al., 2020; Costa Neto et al., 2021a; Crossa et al., 2021).

From envirotyping, it is possible to check the quality of a certain environment, which is directly related to how the observed growing conditions in a particular field trial could be related to the most frequent environment-types (envirotypes) that occur in the breeding program TPE or target region (e.g., Heinemann et al., 2019; Cooper et al., 2021; Antolin et al., 2021). In agricultural research, the quality of a certain environment is directly related to how it can limit the expression of the genetic potential of

58 the certain crop for a certain trait, such as suggested by the movement called 'School of de Wit' since 591965 (see Bouman et al., 1996). Thus, for the plant breeding research, this is also direct factors such 60 as genotype $\times$ environment interaction (e.g., Allard, 1964; Finlay and Wilkinson, 1963) and its 61 implications of how the target germplasm under selection (or testing) can perform across the target 62 growing conditions in which the candidate cultivars will be cropped. 
64 phenotyping is still required (Crossa et al., 2017), although prediction-based tools and simulations can support more comprehensive and faster selection decisions (Galli et al., 2020; Cooper et al., 2021; Crossa et al., 2021). One of the most widely used predictive tools is the whole-genome prediction (GP, Meuwissen et al., 2001), developed and validated for several crop species and application scenarios (Crossa et al., 2017; Voss-Fels et al., 2019), such as the selection among populations and the prediction of the performance of single-crosses across multiple environments. For the latter, the most important use of GP mostly relies on the better use of the available phenotyping records and large-scale easymanaged genomic information to expand the spectrum of evaluated single-crosses in silico (Messina et al., 2018; Rogers et al., 2021). Those phenotypic records (e.g., grain yield and plant height) are collected from existing field trials that experience a diverse set of growing conditions, carrying within them an intrinsic environment-phenotype covariance. Consequently, the GP has a limited accuracy under multiple-environment testing $(\mathrm{MET})$ due to genotype $\times$ environment interaction $(\mathrm{G} \times \mathrm{E})(\mathrm{Crossa}$ et al., 2017), meaning that each genotype has a differential response for each environmental factor that assembles what we call 'environment' (time interval across crop lifetime involving a specific geographic location and agronomic practice for a particular crop). Therefore, novel ways to include environmental data (Heslot et al., 2014; Jarquín et al., 2014; Ly et al., 2018; Millet et al., 2019; Gillberg et al., 2019; Costa-Neto et al., 2021a) and process-based crop growth models (CGM) (Messina et al., 2018; Toda et al. 2020; Robert et al., 2020; Cooper et al., 2021) in GP are considered the best pathways to fix it. Most of the success achieved by such approaches lies in a better understanding of the visible ecophysiology interplay between genomics and environment variation (Gage et al., 2017; Li et al., 2018; Guo et al., 2020; Costa-Neto et al., 2021b). 

al., 2021a), to optimize MET networks and to screen genotype-specific reaction-norms (Ly et al., 2018; Millet et al., 2019). This is excellent progress for predictive breeding (i.e., the range of predictionbased selection tools for crop improvement) and accelerating research pipelines to deliver higher yields and adapted genotypes for target scenarios. However, most of the current studies on this topic vary in accuracy and applicability, mostly due to (1) the processing protocols used to translate the raw-data

93 into explicit environmental covariables (ECs) with biological meaning in explaining G×E over 94 complex traits, (2) the lack of a widely-used envirotyping pipeline that, not only supports the design of field trials, but also increases the accuracy of the trained GP models and, in addition, (3) for CGM, a possible limitation is the increased demand for the phenotyping of additional intermediate phenotypes (i.e., biomass accumulation and partitioning, specific leaf area), which can involve managed isoenvironments and expert knowledge in crop modeling (Cooper et al., 2016; Toda et al., 2020; Robert et al., 2020). The latter can be expensive or difficult for plant research programs in developing countries, which generally have low budgets to increase the phenotyping network and install environmental sensors. In addition, most developing countries are located in regions where environments are subject to a broader range of stress factors (e.g., heat stress). source for predictive breeding, hereafter denominated as enviromic assembly. The benefits of using the so-called 'enviromics-aided GBLUP' (E-GP) under existing experimental networks are presented,

107 followed by the E-GP application to optimize field-based phenotyping. Finally, we benchmark E-GP with the traditional genomic-best unbiased prediction (GBLUP) to discuss the benefits of enviromic data to reproduce $\mathrm{G} \times \mathrm{E}$ patterns and provide a virtual screening for yield plasticity. 


\section{MATERIAL AND METHODS}

112 The material methods are organized in the following manner: First, we briefly address the

113 concepts underlying the novel approach of enviromic assembly inspired by Shelford's Law. The data

114 sets are then presented, along with the statistical models and prediction scenarios used to show the

115 benefits of large-scale environmental information in GP across multi-environment trials (MET).

116 Finally, we present a scheme to optimize phenotyping efforts in training GP over MET and support the

117 screening for maize single-crosses' yield plasticity.

\section{2.1 Theory: adapting the Shelford Law of Minimum}

119 Consider two experimental networks (MET) of the same target population of environments (TPE,

120 e.g., the different locations, years, and crop management) under different environmental gradients due

121 to year or location variations (Fig.1). For two genotypes evaluated under both conditions (G1, G2), the

122 potential genetic-specific phenotypic plasticity (Allard and Bradshaw, 1964) (curves) is expressed as

123 different reaction-norms (dotted lines), resulting in distinct observable G×E patterns (Fig.1a-b). In the

124 former MET (Fig.1a), both genotypes experience a wider range of possible growing conditions (large

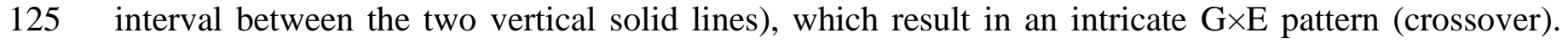

126 Conversely, in the latter MET (Fig.1b), the same genotypes experience a reduced range of growing

127 conditions yet lead to a simple $\mathrm{G} \times \mathrm{E}$ pattern (non-crossover). It is feasible to conclude that, although

128 the genetic variation is essential for modeling potential phenotypic plasticity of genotypes (curves,

129 Fig.1a-c), the diversity of environmental growing conditions dictates the observable G $\times$ E patterns

130 (Bradshaw, 1965). Thus, the GP platforms for MET may be unbiased with no diversity, and the quality

131 of environments is not considered.

132 Approaches such as CGM try to reproduce the phenotypic plasticity curves, while benchmark

133 reaction-norm models try to reproduce the observable reaction-norm. Both approaches can achieve 
134 adequate results, although we have observed that (1) CGM demands greater phenotyping efforts to

135 train computational approaches capable of reproducing the achievable phenotypic plasticity from a

136 reduced core of phenotypic records from field trials at near-iso environments (e.g., well-watered

137 conditions versus water-limited conditions for the same planting date and management), (2) CGM

138 demands additional programing efforts, which, for some regions or crops, can be expensive and limit

139 the applicability of the method, (3) adequate reaction-norm models over well-designed phenotyping

140 platforms are not a reality for certain regions of the world with limited resources to invest in precision

141 phenotyping efforts.

We understand that Shelford's Law of Tolerance (Shelford, 1931) is suitable to explain how the

143 environment drives plant plasticity and can be incorporated into the traditional GP platforms in a cost-

144 effective way (Fig.1c). It states that a target population's adaptation is modulated as a certain range of

145 minimum, maximum and optimum threshold limits achieved over an environment gradient (vertical

146 solid green lines). The genotypes' potential phenotypic plasticity (curves) is not regarded as a linearized

147 reaction-norm variation across an environmental gradient (Arnold et al., 2019). Instead, it is the

148 distribution of possible phenotypic expressions dictated by the cardinal thresholds for each biophysical

149 factor with ecophysiological relevance. Therefore, crops may experience stressful conditions due to

150 the excess or lack of a target environmental factor, depending on the cardinal thresholds (vertical solid

151 green lines in Fig.1c), which also rely on some key development stages germplasm-specific

152 characteristics (e.g., tropical maize versus temperate maize). Consequently, the expected variation of

153 environmental conditions across different field trials results from a series of environment-types

154 (envirotypes) acting consistently yet varying in impact depending on the genetic-specific sensibility.

155 The quality of a certain growing condition depends on the balance between crop necessity and resource

156 availability, which involves constant effects, such as the type of treatments in a trial (e.g., fertilizer

157 inputs) and transitory effects variables, such as weather events (e.g., heat-stress). 
From these concepts, we observe that with the use of envirotyping (e.g., typing the profiles of a

159 particular environment), the environment part of the G $\times E$ pattern can be visualized based on the shared

160 frequency of envirotypes among different field trials. Thus, the enviromic of a certain experimental

161 network or TPE (the core of possible growing conditions) can be mathematically assembled by (1)

162 collecting large-scale environmental data, (2) processing this raw data in envirotyping entries for each

163 real or virtual environment, and (3) processing these envirotyping-derived entries to achieve theoretical

164 relatedness between the buildup of different environments from the shared frequency of envirotypes.

165 Thus, the expected envirotypes can be designed relying on the adaptation zones inspired by the model

166 proposed here, based on Shelford's Law, in which we can envisage the process of deriving

167 environmental covariables for GP into an ecophysiological-smart way.

\subsection{Proof-of-concept data sets}

This study used maize as a proof-of-concept crop due to its importance for food security in

170 developed and developing regions. Two data sets of maize hybrids (single-crosses of inbreed lines)

171 from different germplasm sources developed under tropical conditions in Brazil (hereafter referred to

172 as Multi-Regional and N-level) were used. Both data sets involve phenotypic records of grain yield

173 (Mg per ha) collected across multiple environments. Details on the experimental design, cultivation

174 practices, and fundamental statistical analysis are given in Bandeira e Souza et al. (2017) and Alves et

175 al. (2019). Below we provide a short description of the number of genotypes and environments tested

176 and the nature of this study's genotyping data.

\subsubsection{Multi-Regional Set}

The so-called "Multi-Regional set" is based on the germplasm developed by the Helix Seeds

179 Company (HEL) in South America. It includes 247 maize lines evaluated in 2015 in five locations in

180 three regions of Brazil (Supplementary Table 1). Genotypes were obtained using the Affymetrix Axiom 
181 Maize Genotyping Array containing 616 K SNPs (single-nucleotide polymorphisms) (Unterseer et al.,

182 2014). Only SNPs with a minor allele frequency $>0.05$ were considered. Finally, a total of 52,811

183 high-quality SNPs that achieved the quality control level were used in further analysis.

\subsubsection{N-level set}

The so-called "N-level set" is based on the germplasm developed by the Luiz de Queiroz College

187 evaluated across eight environments, involving an arrangement of two locations, two years, and two

nitrogen levels (Supplementary Table 2). This study's sites involved two distinct edaphoclimatic

189 patterns, i.e., Piracicaba (Atlantic forest, clay soil) and Anhumas (savannah, silt-sandy soil). In each

190 site, two contrasting nitrogen $(\mathrm{N})$ fertilization levels were managed. One experiment was conducted

191 under ideal $\mathrm{N}$ conditions and received $30 \mathrm{~kg} \mathrm{ha}^{-1}$ at sowing, along with $70 \mathrm{~kg} \mathrm{ha}^{-1}$ in a coverage

192 application at the V8 plant stage. That is the main recommendation for fertilization in tropical maize

193 growing environments in Brazil. In contrast, the second experiment under low $\mathrm{N}$ conditions received

194 only $30 \mathrm{~kg} \mathrm{ha}^{-1}$ of $\mathrm{N}$ at sowing, resulting in an $\mathrm{N}$-limited growing condition. This set's genotypes were

195 also obtained using the Affymetrix Axiom Maize Genotyping Array containing 616 K SNPs (Unterseer

196 et al., 2014) and minor allele frequency > 0.05. At the end of this process, a total of 54,113 SNPs were

197 considered in the GP modeling step.

\section{$198 \quad 2.3$ Envirotyping Pipeline}

Below, we present the methods used for data collection, data processing, and implementing what

200 we call 'enviromic assembly'. This envirotyping pipeline was developed using the functions of the R

201 package EnvRtype (Costa-Neto et al., 2021) and is available at no cost. All codes for running the next

202 steps are given in https://github.com/gcostaneto/EGP. 
In this study, environmental information was used for the main abiotic plant-environment interactions related to daily weather, soil type, and crop management (available only for N-level set). variables: rainfall $\left(\mathrm{P}, \mathrm{mm}\right.$ day $\left.^{-1}\right)$, maximum air temperature $\left(\mathrm{TMAX},{ }^{\circ} \mathrm{C}\right.$ day $\left.^{-1}\right)$, minimum air temperature $\left(\mathrm{TMIN},{ }^{\circ} \mathrm{C}\right.$ day $\left.^{-1}\right)$, average air temperature $\left(\mathrm{TAVG},{ }^{\circ} \mathrm{C}\right.$ day $^{-1}$ ), dew point temperature $\left(\mathrm{TDEW},{ }^{\circ} \mathrm{C}\right.$ day $\left.^{-1}\right)$, global solar radiation (SRAD, MJ per $\mathrm{m}^{2}$ ), wind speed at 2 meters $\left(\mathrm{WS}, \mathrm{m} \mathrm{s}^{-1}\right.$ day $^{-}$

$\left.{ }^{1}\right)$ and relative air humidity $\left(\mathrm{RH}, \%\right.$ day $\left.^{-1}\right)$. Elevation above sea level was obtained from NASA's Shuttle environments using the functions and libraries organized within the EnvRtype package (Costa-Neto et al., 2021b). A third GIS database was used to import soil types from Brazilian soil classification provided by EMBRAPA and available at https://github.com/gcostaneto/EGP.

\subsubsection{Data Processing}

Quality control was adopted by removing variables outside the mean \pm three standard deviation and repeated columns. After checking for outliers, the daily weather variables were used to model ecophysiological interactions related to soil-plant-atmosphere dynamics. The thermal-radiation interactions computed potential atmospheric evapotranspiration (ET0) following the Priestley-Taylor method. The slope of the saturation vapor pressure curve (SPV) and vapor pressure deficit (VPD) was computed as given in the FAO manual (Allen et al., 1998). An FAO-based generic function was used to estimate crop development as a function of days after emergence (DAE). We assume a 3-segment leaf growing function to estimate the crop canopy coefficient $(\mathrm{Kc})$ of evapotranspiration using the

224 following $\mathrm{Kc}$ values: $\mathrm{Kc}_{1}(0.3), \mathrm{Kc}_{2}(1.2), \mathrm{Kc}_{3}(0.35)$, equivalent to the water demand of tropical maize 225 for initial phases, reproduction phases, and end-season stages, respectively. Using the same 3-segment 226 function, we estimate the crop canopy using a leaf area index (LAI) of LAI = 0.7 (initial vegetative 
phases), LAI = 3.0 (maximum LAI for tropical maize growing conditions observed in our fields), and

LAI $=2.0$ (LAI tasseling stage). We computed the daily crop evapotranspiration (ETc) estimated by

the product between ET0 and the Kc from those two estimations. Then, we computed the difference between daily precipitation and crop evapotranspiration as P-ETc.

The apparent photosynthetic radiation intercepted by the canopy (aPAR) was computed

$\mathrm{F}_{\mathrm{RUE}}=0$ if $\mathrm{T}_{\mathrm{AVG}} \leq \mathrm{Tb}_{1} ; \mathrm{F}_{\mathrm{RUE}}=\left(\mathrm{T}_{\mathrm{AVG}}-\mathrm{Tb}_{1}\right) /\left(\mathrm{To}_{1}-\mathrm{Tb}_{1}\right)$ if $\mathrm{Tb}_{1}<\mathrm{T}_{\mathrm{AVG}}<\mathrm{To}_{1} ; \mathrm{F}_{\mathrm{RUE}}=1$ if $\mathrm{To}_{1}<\mathrm{T}_{\mathrm{AVG}}<$

$\mathrm{To}_{2} ; \mathrm{F}_{\mathrm{RUE}}=\left(\mathrm{Tb}_{2}-\mathrm{T}_{\mathrm{AVG}}\right) /\left(\mathrm{Tb}_{2}-\mathrm{To}_{2}\right)$ if $\mathrm{To}_{2}<\mathrm{T}_{\mathrm{AVG}}<\mathrm{Tb}_{2} ;$ and $\mathrm{F}_{\mathrm{RUE}}=0$ if $\mathrm{T}_{\mathrm{AVG}}>\mathrm{Tb}_{2}$. stands for days after emergence.

\subsubsection{Enviromic assembly using typologies ( $T$ matrix)}

The raw envirotyping data were used to assemble markers for environmental similarity, 
adaptation: (1) stress by deficit, (2) optimum growing conditions, and (3) stress by excess. These levels

were defined using cardinal thresholds or frequency tables concerning the growing conditions archived

in the experimental network range. Then, from having reviewed the literature, we consider the intervals

253 for thermal-related variables: $0^{\circ} \mathrm{C}$ to $9^{\circ} \mathrm{C}$ (death), $9.1^{\circ} \mathrm{C}$ to $23^{\circ} \mathrm{C}$ (stress by deficit), $23.1{ }^{\circ} \mathrm{C}$ to $32^{\circ} \mathrm{C}$

254 (optimum growing conditions), $32.1^{\circ} \mathrm{C}$ to $45^{\circ} \mathrm{C}$ (stress by excess) and $45^{\circ} \mathrm{C}$ to $\infty^{\circ} \mathrm{C}$ (death). We

255 computed the classes for accumulated prediction according to our agronomic expertise on rainfall

256 requirements for tropical maize growing environments: $0 \mathrm{~mm}$ to $10 \mathrm{~mm}, 10.1 \mathrm{~mm}$ to $20 \mathrm{~mm}$, and $20.1 \mathrm{~mm}$

257 to $\infty \mathrm{mm}$. For crop evapotranspiration (ETc), we assume the envirotypes 0-6 mm.day-1, 7-10 mm.day-

$2581,10-15$ mm.day-1 and 16 to $\infty$ mm.day-1. Finally, for FRUE, we assume the envirotypes based on the

259 following adaptation zones: impact from $0 \%$ to $25 \%(0-0.25)$, from $26 \%$ to $50 \%(0.26-0.50), 51 \%$ to

$26075 \%(0.51-0.75)$ and $76 \%$ to $100 \%(0.76-1.0)$. We preferred to adopt a simple discretization for the

261 remaining variables using a histogram of percentiles $(0-25 \%, 26-50 \%, 51-75 \%, 75-100 \%)$ of

262 occurrence for a target envirotype.

The second group involves constant effect variables. In this group, we consider the elevation,

crop management, and soil classification in each environment. Soil information was entered as an

incidence matrix ( 0 or 1 ) based on each environment's occurrence. In addition, for the N-level set,

266 nitrogen input levels were computed as two discrete classes: ideal $\mathrm{N}=10$ and low $\mathrm{N}=30$; we entered

267 this same incidence matrix for soil information. Because both sets have a gradient for elevation, we

268 used a histogram of percentiles $(0-25 \%, 26-50 \%, 51-75 \%, 75-100 \%)$ as in the transitory group of

variables. Finally, each designed envirotype $\times$ time interval frequency was used as a qualitative marker

270 of environmental relatedness (the hereafter $\mathbf{T}$ matrix, from typologies). 
The quantitative descriptors of environmental relatedness are the most common method to

273 include environmental information in GP studies considering reaction-norms. Jarquín et al. (2014)

274 proposed the creation of the so-called environmental relatedness kinship $\left(\boldsymbol{K}_{\mathrm{E}}\right)$ carried out with a matrix

275 of quantitative environmental covariables ( $\mathbf{W}$ matrix, thus we refer to this environment kinship as

$\left.276 \boldsymbol{K}_{\mathrm{E}, \mathrm{W}}\right)$. Here, this pattern of similarity in $\boldsymbol{K}_{\mathrm{E}, \mathrm{W}}$ was captured using percentile values $(25 \%$; $50 \%$, and

$27775 \%$ ) at each of the five-time intervals of development, as suggested by Morais-Júnior et al. (2018)

278 and expanded by Costa-Neto et al., (2021a). We found 255 and 307 quantitative descriptors for the

279 Multi-Regional and N-level sets at the end of the process, respectively. In this study, we used this $\boldsymbol{K}_{\mathrm{E}, \mathrm{W}}$

280 as a benchmark method to test the effectiveness of the $\boldsymbol{K}_{\mathrm{E}, \mathrm{T}}$ matrix and the total absence of

281 environmental information (baseline genomic model without environmental information, see section

$282 \quad 2.4 .1)$.

\section{$284 \quad 2.4$ Statistical Models}

From a baseline additive-dominant multi-environment GBLUP (section 2.4.1), we tested four

other models, created with the inclusion of two types of enviromic assembly ( $\mathbf{T}$ or $\mathbf{W}$ ) and structures

for $\mathrm{G} \times \mathrm{E}$ effects. More details about each statistical model are provided in the next subsections. All

288 kernel models were fitted using the BGGE R package (Granato et al., 2018) using 15,000 iterations,

289 with 2,000 used as burn-in and using a thinning of 10. This package was used due to the following

290 aspects: (1) is an accurate open-source software and; (2) can accommodate many kernels in a

291 computation-efficient way. 

overall main effect plus a genomic-by-environment deviation (the so-called G+GE model, Bandeira e Souza et al., 2017), as follows:

$$
\boldsymbol{y}=\mathbf{1} \boldsymbol{\mu}+\boldsymbol{Z}_{E} \boldsymbol{\beta}+\boldsymbol{Z}_{A} \boldsymbol{u}_{A}+Z_{D} \boldsymbol{u}_{D}+\boldsymbol{u}_{A E}+\boldsymbol{u}_{D E}+\boldsymbol{\varepsilon}
$$

298

where $\boldsymbol{y}=\left[\boldsymbol{y}_{1}, \cdots, \boldsymbol{y}_{\boldsymbol{n}}\right]^{\prime}$ is the vector of observations collected in each of the $q$ environments with hybrids and $\mathbf{1} \boldsymbol{\mu}+\boldsymbol{Z}_{\mathrm{E}} \boldsymbol{\beta}$ is the general mean and the fixed effect of the environments with incidence matrix $\boldsymbol{Z}_{E}$. Genetic variations are modeled using the main additive effects $\left(\boldsymbol{u}_{\mathrm{A}}\right)$, with $\boldsymbol{u}_{\mathrm{A}} \sim$

304 incidence matrix for the same effects (absence $=0$, presence $=1$ ), $J_{q}$ is a $q \times q$ matrix of $1 \mathrm{~s}$ and $\otimes$ denotes

$$
\mathbf{D}=\left\{-2 f_{l}^{2}=A^{2} A^{2} ; 2 f\left(1-f_{l}\right)=A^{1} A^{2} ;-2 f\left(1-f_{l}\right)^{2}=A^{1} A^{1}\right\},
$$

312 where $f_{l}$ is the frequency of the favorable allele at locus $l$. Thus, the genomic-related kinships were 313 estimated as follows: 


$$
\boldsymbol{K}=\frac{\boldsymbol{X} \boldsymbol{X}^{\prime}}{\operatorname{trace}\left(\boldsymbol{X} \boldsymbol{X}^{\prime}\right) / \operatorname{nrow}(\boldsymbol{X})}
$$

315 where $\boldsymbol{K}$ is a generic representation of the genomic kinship $\left(\boldsymbol{K}_{\mathrm{A}}, \boldsymbol{K}_{\mathrm{D}}\right), \boldsymbol{X}$ is a generic representation of

316 the molecular matrix (A or $\mathbf{D})$, and $\operatorname{nrow}(\boldsymbol{X})$ denotes the number of rows in $\boldsymbol{X}$ matrix. Eq (2) was also

317 used to shape the environmental relatedness kernels using T or W matrix. This linear kernel for $\mathbf{K}_{E}$ was

318 described by Jarquín et al. (2014), which some other authors named it after " $\mathbf{\Omega}$ ". Thus, here we only

319 tested the difference between the enviromic source considered for building it and not the merit of the

320 kernel method as was done in previous works (Costa-Neto et al., 2021a).

2.4.2GBLUP with enviromic main effects from $T$ matrix ( $E-G P)$

From baseline equation (1), we include a main environmental relatedness effect carried out with

$$
\boldsymbol{y}=\mathbf{1} \boldsymbol{\mu}+\boldsymbol{Z}_{A} \boldsymbol{u}_{A}+\boldsymbol{Z}_{D} \boldsymbol{u}_{D}+\boldsymbol{u}_{A E}+\boldsymbol{u}_{D E}+\boldsymbol{u}_{E, T}+\boldsymbol{\varepsilon}
$$

327 with $\boldsymbol{u}_{\mathrm{E}, \mathrm{T}} \sim N\left(\boldsymbol{Z}_{E} \boldsymbol{\beta}, \boldsymbol{K}_{\mathrm{E}, \mathrm{T}} \otimes \boldsymbol{J}_{p} \boldsymbol{\sigma}_{\mathrm{E}, \mathrm{T}}^{2}\right)$, where $\boldsymbol{J}_{q}$ is a $p \times p$ matrix of $1 \mathrm{~s}$, is $\boldsymbol{K}_{\mathrm{E}, \mathrm{T}}$ the environmental

328 relatedness created and variance component from the $\mathbf{T}$ matrix. If non-enviromic sources are

329 considered, the expected value for environments is given by $\boldsymbol{Z}_{E} \boldsymbol{\beta}$ as the baseline model. In this model,

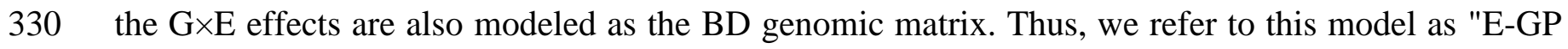

331 (BD)". The kernel of enviromic assembly $\left(\boldsymbol{K}_{E, T}\right)$ was built using the panel of envirotype descriptors

332 (T) in the same way as described in equation (2).

333 From model (3), we substitute the BD for a reaction-norm (RN, Jarquín et al., 2014) based on

334 the Kronecker product between the enviromic and genomic kinships (Martini et al., 2020) for additive

$335\left(\boldsymbol{u}_{A E, T}\right)$ and dominance effects $\left(\boldsymbol{u}_{D E, T}\right)$ : 


$$
\boldsymbol{y}=\mathbf{1} \boldsymbol{\mu}+\boldsymbol{Z}_{A} \boldsymbol{u}_{A}+\boldsymbol{Z}_{D} \boldsymbol{u}_{D}+\boldsymbol{u}_{T}+\boldsymbol{u}_{A, T}+\boldsymbol{u}_{D, T}+\boldsymbol{\varepsilon}
$$

339 with $\boldsymbol{u}_{\mathrm{A}, \mathrm{ET}} \sim N\left(\mathbf{0}, \boldsymbol{K}_{\mathrm{E}, \mathrm{T}} \otimes \boldsymbol{K}_{A} \boldsymbol{\sigma}_{\mathrm{AE}, \mathrm{T}}^{\mathbf{2}}\right)$ and $\boldsymbol{u}_{\mathrm{D}, \mathrm{ET}} \sim N\left(\mathbf{0}, \boldsymbol{K}_{\mathrm{E}, \mathrm{T}} \otimes \boldsymbol{K}_{D} \boldsymbol{\sigma}_{\mathrm{DE}, \mathrm{T}}^{2}\right)$ where $\boldsymbol{\sigma}_{\mathrm{AE}, \mathrm{T}}^{2}$ and $\boldsymbol{\sigma}_{\mathrm{DE}, \mathrm{T}}^{2}$ are

340 the variance components for enviromic $\times$ additive and enviromic $\times$ dominance effects performed as

341 reaction-norms (Costa-Neto et al., 2021a; Rogers et al., 2021), respectively. For short, this model will

342 be named "E-GP (RN)".

343 2.4.3 GBLUP with enviromic main effects from $W$ matrix ( $W$-GP)

344 Finally, in models (4) and (5), we substitute the enviromic assembly derived from $\mathbf{T}$ by the

345 same kernel size derived from $\mathbf{W}$, that is, an environmental relatedness with $\boldsymbol{u}_{\mathrm{E}, \mathrm{W}} \sim N\left(\boldsymbol{Z}_{E} \boldsymbol{\beta}, \boldsymbol{K}_{\mathrm{E}, \mathrm{W}} \otimes\right.$

$346 \boldsymbol{J}_{p} \boldsymbol{\sigma}_{\mathrm{E}, \mathrm{W}}^{2}$, creating two more models:

347

$$
\boldsymbol{y}=\mathbf{1} \boldsymbol{\mu}+\boldsymbol{Z}_{A} \boldsymbol{u}_{A}+\boldsymbol{Z}_{D} \boldsymbol{u}_{D}+\boldsymbol{u}_{A E}+\boldsymbol{u}_{D E}+\boldsymbol{u}_{E, w}+\boldsymbol{\varepsilon}
$$

349 and

$$
\boldsymbol{y}=\boldsymbol{1} \boldsymbol{\mu}+\boldsymbol{Z}_{A} \boldsymbol{u}_{A}+\boldsymbol{Z}_{D} \boldsymbol{u}_{D}+\boldsymbol{u}_{E, w}+\boldsymbol{u}_{A E, w}+\boldsymbol{u}_{D E, w}+\boldsymbol{\varepsilon}
$$

$\boldsymbol{u}_{\mathrm{AE}, \mathrm{W}} \sim N\left(\mathbf{0}, \boldsymbol{K}_{\mathrm{E}, \mathrm{W}} \otimes \boldsymbol{K}_{A} \boldsymbol{\sigma}_{\mathrm{AE}, \mathrm{W}}^{2}\right)$ and $\boldsymbol{u}_{\mathrm{DE}, \mathrm{W}} \sim N\left(\mathbf{0}, \boldsymbol{K}_{\mathrm{E}, \mathrm{W}} \otimes \boldsymbol{K}_{D} \boldsymbol{\sigma}_{\mathrm{ED}, \mathrm{W}}^{2}\right)$, where $\boldsymbol{K}_{\mathrm{E}, \mathrm{W}}$ and $\boldsymbol{\sigma}_{\mathrm{E}, \mathrm{W}}^{2}$ are

353 the resulting kinship and the variance components estimated for enviromic assembly from the $\mathbf{W}$

354 matrix, respectively. Thus, for short, models (5) and (6) will be referred to as "W-GP (BD)" and "W-

355 GP (RN)", respectively. 


\subsection{Study cases for the E-GP platform}

In this study, we conceived two cases to highlight the benefits of E-GP to boost efficiency in

359 prediction-based platforms for hybrid development in maize breeding (Figure 2). The first case (Case

360 1) involves predicting the single-crosses from different theoretical existing experimental network

361 setups, where we dissect the predictive ability over four prediction scenarios. In the second case (Case

362 2), we explore a theoretical conception of a super-optimized experimental network using the most

363 representative combination of genotypes-environments selected using genomics, enviromic assembly,

364 and genetic algorithms. Below we detail each case studied.

\subsubsection{Case 1: expanding the existing field trials}

In the first case (Case 1), we design a novel cross-validation scheme to split the global available phenotypic information $(n)$, from $p$ genotypes and $q$ environments, into different training setups.

368 Consequently, four prediction scenarios were created based on the simultaneous sampling of the phenotypic information for $S$ genotypes and $R$ environments.

- $\mathrm{G}, \mathrm{E}$ : refers to the predictions of the tested genotypes within the experimental network (known genotypes in known environmental conditions). The size of this set is $\mathrm{n}_{[\mathrm{G}, \mathrm{E}]}=\mathrm{n} \times\left(\frac{\mathrm{S}}{\mathrm{p}}\right) \times\left(\frac{\mathrm{R}}{\mathrm{q}}\right)$;

- $n \mathrm{G}, \mathrm{E}$ : refers to predictions of untested (new) genotypes within the experimental network (known

373 environmental conditions). The size of this set is $\mathrm{n}_{[\mathrm{nG}, \mathrm{E}]}=\mathrm{n} \times\left(1-\frac{\mathrm{s}}{\mathrm{p}}\right) \times\left(\frac{\mathrm{R}}{\mathrm{q}}\right)$;

- $\mathrm{G}, n \mathrm{E}$ : in this scenario, predictions are made under environmental conditions external to those found within the experimental network. However, there is phenotypic information available within the experimental network. The size of this set is $n_{[G, n E]}=n \times\left(\frac{S}{p}\right) \times\left(1-\frac{R}{q}\right)$; 
- $n \mathrm{G}, n \mathrm{E}$ : refers to predicting untested (new) genotypes and untested (new) environmental conditions.

the training set for all experimental setups, equal to a fraction of $\frac{S}{p}=0.7$. Each training setup was ability to replace field trials, we then computed the coincidence (CS, in \%) between the field-based selection and the selection-based selection of the top 5\% best-performing hybrids in each environment.

\subsubsection{Case 2: designing super-optimized field trials}

The second case (Case 2) was performed on the optimized training set described below. The first step was to compute a full-entry G×E kernel, based on the Kronecker product $(\otimes)$ between the enviromic assembly-based relatedness $\operatorname{kernel}\left(\boldsymbol{K}_{E, T}, q \times q\right.$ environments $)$ and genomic kinship $\left(\boldsymbol{K}_{G}, p\right.$ $\times p$ genotypes), thus $\boldsymbol{K}_{G E, T}=\boldsymbol{K}_{E, T} \otimes \boldsymbol{K}_{G}$, with an $n \times n$ dimension, in which $n=p q$. Here we adopted

the kernel made up for additive effects $\left(\boldsymbol{K}_{G}=\boldsymbol{K}_{A}\right)$ as the genomic kinship, despite the benefits of 
Alves et al., 2019; Costa-Neto et al., 2021a; Roger et al., 2021), a fact that was also observed for Case 1 (see section 3.1). Later, we applied a single-value decomposition in $\boldsymbol{K}_{G E, T}$, following $\boldsymbol{K}_{G E, T}=\boldsymbol{U V} \boldsymbol{U}^{\boldsymbol{T}}$ where $\boldsymbol{U}$ is a total of eigenvalues and $\boldsymbol{V}$ the respective eigenvectors. The number of eigenvalues that

402 explains $98 \%$ of the variance present in $\boldsymbol{K}_{G E, T}$ indicate the number of effective SNPs by envirotype-

403 marker interactions, which is also the minimum core of genotype-environment combinations $\left(N_{G E}\right)$.

404 Thus, the reduced phenotypic information of some genotypes in some environments $\left(N_{G E}\right)$ was used 405 to predict a virtual experimental network $\left(N_{\text {test }}\right)$, involving all remaining single-crosses in all available 406 environments, thus given by $N_{\text {test }}=n-N_{G E}$,

407 Following this step, a genetic algorithm scheme using the design criteria PEV MEAN was used to identify 408 the $N_{G E}$ combinations of genotypes in environments within the $\boldsymbol{K}_{G E, T}$ entries that must be phenotyped 409 (Misztal, 2016). This optimization was implemented using the SPTGA R package (Akdemir and Isidro410 Sánchez, 2019) using 100 iterations: five solutions selected as elite parents were used to generating the 411 next set of solutions and mutations of $80 \%$ for each solution generated.

\section{$412 \quad 2.6$ Virtual screening for yield plasticity}

413 Finally, we tested each GP model's potentials to predict the genotypes' phenotypic plasticity and 414 stability across environments using only the $N_{G E}$ phenotypic information. First, the prediction ability

415 was computed for genotypes by correlating the predicted and observed grain yield values across 416 environments (Costa-Neto et al., 2021a). The second measure was based on the Finlay-Wilkinson 417 adaptability model's regression slope (Finlay and Wilkinson, 1963). The GP predicted values were 418 regressed to the observed environmental deviations, as follows:

$$
M_{i j}=\bar{y}_{i .}+b_{i} I_{j}+\varepsilon_{i j}
$$


420 where $M_{i j}$ is the expected GP-based mean value of grain yield for $i^{\text {th }}$ genotype at $j^{\text {th }}$ environment, $\bar{y}_{i}$ is

421 the mean genotypic value for $i^{\text {th }}$ genotype, $b_{i}$ is the genotype plastic response across the mean-centered

422 standardized environmental score $\left(I_{j}\right)$ and $\varepsilon_{i j}$ is the variety of residual deviation sources not accounted

423 in the model. After this step, the Pearson's product-moment correlation between GP-based $\left(\hat{b}_{i}\right)$ and

424 phenotypic-enabled estimates were computed as an indicator of the ability to reproduce plastic

425 responses in silico for the $p$ genotypes. For this, the mean squared error is also calculated as:

426

$$
M S E=\sum_{i=1}^{p} \frac{\left(b_{i}-\hat{b}_{i}\right)^{2}}{p}
$$

427 All statistics were computed using the entire data sets and only the top 5\% of genotypes selected for

428 each environment. The latter aimed to check the efficiency of the E-GP method to produce high-quality

429 virtual screenings for plasticity.

\section{$430 \quad 2.7$ Data and Code availability}

431 All data sets and codes (in R) are freely available at https://github.com/gcostaneto/EGP.

\section{RESULTS}

\subsection{Case 1: Accuracy in predicting diverse G×E scenarios}

A cross-validation scheme was designed to assess the predictive ability of the enviromic-aided approaches in the face of traditional GBLUP. For that, sample genotypes (70\%) and environments were used to compose a drastically sparse training set for MET (training environments/total of

437 environments). This helped assess the efficiency of E-GP for Case 1, in which we were able to dissect

438 the predictive ability (section 3.2.3) in different scenarios of a scarcity of phenotypic records: novel 439 genotypes in tested environments $(n \mathrm{G}, \mathrm{E})$; tested genotypes in untested environments $(\mathrm{G}, n \mathrm{E})$, and novel 
440 genotype and environment conditions $(n \mathrm{G}, n \mathrm{E})$. Tables 1 and 2 present the N-level and Multi-Regional

441 sets results, respectively. Then, these results were gathered for both data sets and four prediction

442 scenarios in order to check for the joint predictive ability analysis (Figure 3).

\subsubsection{Within experimental network (know growing conditions)}

The predictions within known environmental conditions of a certain experimental network

445 involve scenarios $G, E$ and $n G, E$. For the $G, E$ scenario (classical 'training set'), all models

446 outperformed the GBLUP in any setups N-level set, and most of the setups of Multi-Regional set. The

447 highest values of predictive ability were observed for enviromic-aided GP models using the block-

448 diagonal matrix for $\mathrm{G} \times \mathrm{E}$ effects $(\mathrm{BD})$, that is, the E-GP (BD) and W-GP (BD), respectively. Two

449 general trends were observed: the size of the experimental setup has a small effect on GP models'

450 accuracy. Secondly, higher accuracy gains were observed for the N-level set (Table 1), with a higher

451 number of entries (more genotypes and more environments). The accuracy gains in this $\mathrm{N}$ level set

452 ranged from $+8 \%$ ( $r=0.83$ for E-GP RN at 7/8 experimental setup), in relation to $r=0.77$ (GBLUP),

453 to $+24 \%$ ( $r=0.92$ for W-GP RN at $3 / 8$ experimental setup), in relation to $r=0.74$ (GBLUP). In

454 contrast, for the Multi-Regional set (Table 2), both RN-G×E models reduced the accuracy (on average,

$455-3 \%$ ). For the BD-G×E models, small gains in accuracy (from $+4 \%$ to $+8 \%$ ) were observed.

That is also a trend for the second prediction scenario $(n \mathrm{G}, \mathrm{E})$, in which the Multi-Regional set

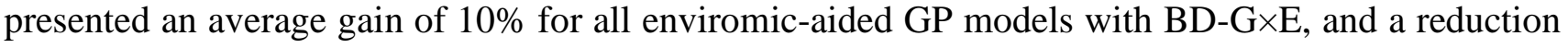

of $10 \%$ for all $\mathrm{RN}-\mathrm{G} \times \mathrm{E}$ models. Conversely to the previous scenario (G,E, within the experimental network, using known genotypes), the $n \mathrm{G}, \mathrm{E}$ is one of the most important plant breeding scenarios. It

461 genomic and enviromic information from the phenotypes of the relatives. Thus, expand the spectrum

462 of possible genotypes using know growing conditions from the past. For the N-level set, gains up to 
$463100 \%$ were observed for all enviromic-aided models using any $\mathrm{G} \times \mathrm{E}$ structure. No differences were

464 observed between enviromic-aided models and experimental setups. On average, all enviromic-aided

465 models achieved a predictive ability of approximately $r=0.66$ across all experimental setups $(3 / 8,5 / 8$,

466 and 7/8, Table 1). In contrast, the GBLUP model has been impacted with reduced accuracy and a lack

467 of phenotypic records. The highest gains in predictive ability were observed for scenario $3 / 8$, average

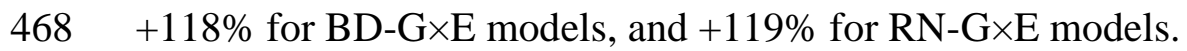

\subsubsection{Across experimental network (new growing conditions)}

The predictions within new environmental conditions across the experimental network involve

$G, n E$ and $n G, n E$. Both scenarios represent the ability of using the available phenotype information

collected from experimental network in order to predict novel growing conditions using genomic or

genomic+enviromic data sources. For the $G, n E$, the E-GP models outperformed W-GP and GBLUP across most experimental setups, despite small differences between the enviromic-aided approaches.

For the E-GP BD at the N-level set (Table 1), the gains in predictive ability ranged from $+24 \%(r=$

to $r=0.43$ (GBLUP). However, for scenario $3 / 8$, these gains were equal to $+10 \%(r=0.57)$ in relation

to the $+13 \%$ archived by the benchmark W-GP RN $(r=0.58)$, both over the $r=0.53$ from GBLUP. In scenario 7/8, W-GP was outperformed by GBLUP, with a reduction in accuracy between -18\% and -

$48016 \%$, where the E-GP made better use of the large phenotypic information available for training GP

481 models (gains from $+20 \%$ to $+24 \%$ over GBLUP). A similar pattern was observed for the Multi-

482 Regional set (Table 2), in which the gains of E-GP ranged from $+4 \%$ to $+6 \%$ across all setups, and $\mathrm{W}$ -

483 GP ranged from $-3 \%$ to $+6 \%$ under the same conditions.

The second scenario involving novel growing conditions also predicts novel genotypes $(n G, n E)$ 
and considering the environmental similarity conceived from enviromics. With a large experimental network and genomics, the E-GP models outperformed W-GP and GBLUP when predicting new G×E. Observed accuracy gains ranged from $33 \%(r=0.39$ for E-GP RN) to $40 \%(r=0.42$ for E-GP BD), in experimental setup 7/8 (Table 1), where GBLUP achieved $r=0.30$, and from $47 \%(r=0.46$ for E-GP BD) to $51 \%$ ( $r=0.48$ for E-GP BD), at the experimental setup 5/8, where GBLUP achieved $r=0.32$.

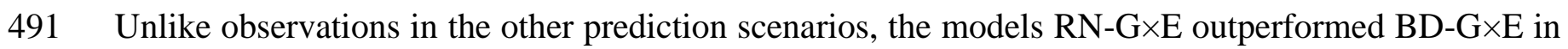
experimental setups 3/8 (N-Level set) and 2/5 (Multi-Regional set).

\subsection{Accuracy trends across diverse experimental setups}

This section highlights the main target of our Case 1 study, in which the predictive ability was achieved using the merged information of scarce genotypes at some environments. Joint accuracy trends showed that E-GP was useful at increasing GP accuracy (Fig.3a) and explaining the phenotypic

phenotypic information (e.g., 3/5, 3/8, and 4/8), any model with some degree of environmental information outperformed the GBLUP for all scenarios. The E-GP approach (purple colors in Figure

3a) better captured envirotype-phenotype relations and converted them into accuracy gains among reproducing field-based trials (Fig.3b).

505 is substantial for most cases E-GP is enough to increase accuracy in GBLUP. For setup 2/5 (Multi506 Regional Set), no differences were observed between all the GP models. 
$40 \%$ for GBLUP (without environmental information). For the E-GP approach accounting for a wide

510 number of phenotypic records in the training set $(7 / 8,3 / 5$, and $4 / 5)$, values of CS up to $55 \%$ were found.

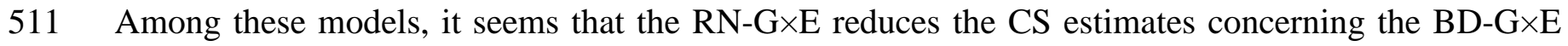

512 based models. Considering both figures $3 \mathrm{a}$ and $3 \mathrm{~b}$, it is possible to suggest that predictive ability does

513 not imply an increase of CS, that is, in the power of selecting the best performing genotypes in certain

514 environments. However, the drastic increase in the E-GP accuracy in relation to the other models leads

515 us to infer that despite the lower rise in CS, the E-GP models are useful when predicting GE for a vast 516 number of single-crosses.

\subsection{Case 2: enviromic assembly with optimized training sets for genomic prediction}

Those results lead us to investigate Case 2 (Fig.2), where we checked the possibility of training

519 efficient and biologically accurate GP scenarios from super-optimized training sets. Then, we checked

520 the potential of using these optimized field trials for predicting novel $\mathrm{G} \times \mathrm{E}$ under the so-called "virtual

521 experimental networks". This approach were implemented by combining two selective phenotyping

522 approaches (Misztal, 2016; Akdemir and Isidro-Sánchez, 2019), aiming to identify combinations of

523 genotypes and environments using in-silico representations of the enviromic assembly $\times$ genomic

524 kinships.

\section{3.3.1 Predicting $\mathrm{G} \times \mathrm{E}$ at virtual experimental networks}

The process of designing virtual networks in maize hybrid breeding involved two steps

527 (Supplementary Fig 1). First, we used a single-value decomposition (SVD)-based algorithm to select

528 the effective number of individuals $\left(N_{\mathrm{GE}}\right)$ (Misztal, 2016) representing at least $98 \%$ of the variation of

$529 \boldsymbol{K}_{G, E T}$. It was done in $\boldsymbol{K}_{G, E T}$ because this kernel represents an in-silico representation of envirotypes

530 and genotypes (Akdemir and Isidro-Sánchez, 2019). Under sparse MET conditions, it led to a training 
531 size equal to $N_{\mathrm{GE}}=67$ and $N_{\mathrm{GE}}=49$ for the $\mathrm{N}$-level set $(n=4,560)$ and Multi-Regional set $(n=1,235)$,

532 respectively. It represents only $1.5 \%$ and $4 \%$ of the whole experimental network; Supplementary Fig.

533 2-3. For didactic purposes, from here onwards, we will represent the values of $N_{\mathrm{GE}}$ as the training set

534 size/number of genotypes.

535 We also checked the use of all environments, although the accuracy differences were tiny in relation

536 to this sparse MET scenario (Table 3). Furthermore, small differences were achieved by E-GP and W-

537 GP models with BD-G×E, but both higher than RN-G×E and GBLUP (Fig 4). Major differences were

538 highlighted as follows:

539 - For within-field trials, predictive ability ranged from $r=0.76$ (W-GP) to $r=0.87$ (E-GP);

540 - For virtual-networks, it ranged from $r=0.14 \pm 0.11$ (GBLUP) to $r=0.60 \pm 0.06$ (E-GP);

541 - In virtual-networks, the predictive ability of models trained with drastically reduced phenotypic

542 records ranged from $r=0.10\left(\mathrm{GBLUP}, \mathrm{N}_{\mathrm{GE}}=67 / 4560\right)$ to $r=0.58\left(\mathrm{E}-\mathrm{GP}, \mathrm{N}_{\mathrm{GE}}=67 / 4560\right)$ and $r=0.18\left(\mathrm{GBLUP}, \mathrm{N}_{\mathrm{GE}}=49 / 1235\right)$ to $r=0.81\left(\mathrm{E}-\mathrm{GP}, \mathrm{N}_{\mathrm{GE}}=49 / 1235\right)$.

544 The predictive ability was computed considering only the top $5 \%$ of genotypes in each environment

545 and data set. The objective was to verify if the GP approaches could adequately predict the performance

546 of the best-evaluated genotypes in the field. For the Multi-Regional set, the predictive ability ranged

547 from $r=0.098\left(\mathrm{GBLUP}, \mathrm{N}_{\mathrm{GE}}=210 / 1235\right)$ to $r=0.579\left(\mathrm{~W}-\mathrm{GP} \mathrm{BD}, \mathrm{N}_{\mathrm{GE}}=49 / 1235\right)$ and $r=0.578(\mathrm{E}-$

548 GP BD, $\left.\mathrm{N}_{\mathrm{GE}}=49 / 1235\right)$; For the N-level set, W-GP outperformed E-GP, leading to $r=0.554(\mathrm{~W}-\mathrm{GP}$

$\left.549 \mathrm{BD}, \mathrm{N}_{\mathrm{GE}}=536 / 4560\right)$ in front of $r=0.554\left(\mathrm{E}-\mathrm{GP} \mathrm{RN}, \mathrm{N}_{\mathrm{GE}}=67 / 4560\right)$ but with less phenotyping data.

550 In contrast, the best E-GP model at the higher number of genotypes and environments evaluated in the

551 field $r=0.484$ (E-GP RN, $\left.\mathrm{N}_{\mathrm{GE}}=536 / 4560\right)$ were outperformed by the same model, yet with less

552 phenotyping data $r=0.554\left(\mathrm{E}-\mathrm{GP} \mathrm{RN}, \mathrm{N}_{\mathrm{GE}}=67 / 4560\right)$. For GBLUP, the effective size of the training 553 set was important, ranging in predictive ability from $r=0.070\left(\mathrm{~N}_{\mathrm{GE}}=67 / 4560\right)$ to $r=0.152\left(\mathrm{~N}_{\mathrm{GE}}=\right.$ 
554 536/4560). The result of both sets suggests that when using enviromic-aided approaches, the use of

555 fewer amounts of, but more representative, phenotyping information is better than more amounts of,

556 yet less representative, phenotyping data.

Figure 4 was created using the average values of Table 3 . This figure shows that the optimization

was more effective for growing conditions contrasting across macro-regions (Fig. 4a) than for

experimental networks involving fewer locations (Fig. 4b). Notably, it is possible to drastically reduce

560 field costs for experimental networks conducted across diverse locations. However, for screening

561 management conditions, greater precautions must be considered with the use of E-GP.

\subsubsection{Predicting genotype-specific plasticity and environmental quality}

In this step, we checked these models' ability to produce virtual screenings for yield plasticity

(Fig.5). We used the Finlay-Wilkinson method (FW, Eq. 7) over the predicted GY means of each

565 genotype $i$ in environment $j\left(M_{i j}\right)$. Hence, we compared the ability of E-GP in the prediction of: (1)

566 individual genotypic responses across environments, (2) the gradient of environmental quality $\left(h_{j}\right)$,

and (3) the plasticity coefficient $\left(b_{1}\right)$ of the FW model describing the rate of responsiveness to $h$. The

568 results in Fig 5 involves a joint analysis of both data sets.

All models that included some degree of enviromic assembly outperformed the GBLUP-based approach when predicting individual genotype responses across the MET (Fig 5a). The median values

571 of $r$ ranged from $r=0.17$ (GBLUP), in which $45 \%$ of the genotypes were not well predicted (red colors),

572 to $r=0.83$ (E-GP), in which up to $60 \%$ of the genotypes were very well predicted (purple colors). The

573 inclusion of any enviromic assembly and $\mathrm{G} \times \mathrm{E}$ structure led to drastic gains in accuracy for a particular

574 genotype response across contrasting (and unknown) G×E conditions (gains up to 378\%). However,

575 the BD structure outperformed RN in terms of resolution (many purple colors in Fig 5a). A major part 
of the accurately predicted performance of genotypes across environments ranged from $r=0.75$ to $r$

$577=1.0$. Due to this, for the next figures, we plotted only the E-GP considering the BD-G $\times$ E structure.

583 linear responsiveness over the environmental variation. The graphical representation of genotype-

584 specific linear reaction norms dictated by the linear regression slope $\left(b_{1}\right)$ was likely more similar to E-

585 GP than GBLUP about those observed in field-based testing (Fig 5b). The accuracy for $b_{1}$ ranged from $r=0.08$ (GBLUP) to $r=0.43(\mathrm{E}-\mathrm{GP})$, an increase of $437 \%$.

\section{DISCUSSION}

agricultural research and modern breeding program routines. We demostrated that enviromics is the science capable of bringing together environment information and quantitative genomics into an ecophysiology-smart manner. In this study, we presented the first report on (1) the use of Shelford's

592 Law to guide the assembly of the enviromics for predictive breeding purposes over experimental

593 networks; (2) the integration of enviromic assembly-based kernels with genomic kinship into 594 optimization algorithms capable of designing selective phenotyping strategies and (3) a break of the paradigm relying on the fact that phenotyping a higher number of genotypes at higher number of environments do not always contribute to increasing the accuracy of GP for contrasting G×E scenarios, but there are pieces of evidences suggesting that enviromics increases accuracy under sparse multi-

598 environment networks; (4) report that the process of deriving markers of environmental relatedness, 
here named 'enviromic assembly', is crucial for the implementation of low-cost GP platforms over

600 multi-environmental conditions.

In this study, we also envisage that the process of enviromic assembly is supported by a strong

602 theoretical background in ecophysiology, illustrating the potential uses of environmental information

603 to increase the accuracy of predictive breeding for yield and plasticity. Our results indicate that the E-

604 GP platform (Figure 2) can fit two types of prediction scenarios in plant breeding programs: (1) better 605 use of the available phenotypic records to train more accurate GP models capable of aiding the selection of genotypes across multi-environmental conditions and (2) a method that reduces costs for field-based testing and enables an early screening for yield plasticity under crossover $\mathrm{G} \times \mathrm{E}$ conditions.

608 Furthermore, we show that any model with some degree of enviromic assembly (by typology or 609 quantitative descriptors) is always better to reproduce the genotypes' environmental quality of field 610 trials and phenotypic plasticity.

611 Below we discuss the aspects that support the use of E-GP for multi-environment predictions,

612 involving the importance of breaking the paradigm that states that enviromics are not necessary to

613 predict $\mathrm{G} \times \mathrm{E}$ accurately. We then discuss how the genomic and enviromic sources are linked in the

614 phenotypic records collected from the fields and how this type of knowledge can improve the quality

615 of the prediction-based pipelines for crop improvement. Finally, we envisage possible environmental-

616 assembly applications supporting other predictive breeding fields, such as optimizing crop modeling

617 calibration and how it can couple a novel level of climate-smart solutions for crop improvement as 618 anticipating the plasticity of a large number of genotypes using reduced phenotypic data.

619 4.1 Why are enviromics important for multi-environment genomic prediction? 
622 Bernardo, 2009; Windhausen et al., 2012; Zhao et al., 2012; Zhang et al., 2015). Under these

623 conditions, the micro-environmental variations within breeding trials (e.g., spatial gradients in soil

624 properties) are minimized in the phenotypic correction step by separating useful genetic patterns and

625 experimental noises (non-genetic patterns). However, those phenotypic records carry the indissoluble

626 effects of macro-environmental fluctuations of certain weather and soil factors that occurred during

627 crop growth and development (Li et al., 2018; Vidotti et al., 2019; Millet et al., 2019; Guo et al., 2020;

628 Jarquín et al., 2020). That seems to be of no concern when predicting novel genotypes under these

629 same growth conditions (the CV1 scheme for single-environment models) yet becomes noise for multi-

630 environment prediction scenarios. It is a consequence of the macro-environment fluctuations in the

631 lifetime of the crops (Allard and Bradshaw, 1964; Bradshaw, 1965; Arnold et al., 2019), responsible

632 for modulating the rate of gene expression (e.g., Jończyk et al., 2017; Liu et al., 2020) and fine-tuning

633 epigenetic variations and related to transcriptional responses (e.g., Vendramin et al., 2020; Cimen et

634 al., 2021).

635 For each unit that we call "environment" (field trial at the specific year, location, planting date,

636 and crop management), there are various environmental factors such as water availability, canopy

637 temperature, global solar radiation, and nutrient content in the soil. The expression of some genotype

638 in some phenotype is then limited by the certain key environmental factors, acting in different levels

639 of crop development as preconized by School of de Wit' since 1965 (see Bouman et al., 1996).

640 However, we revisited the Shelford's theory, which suggests that a population's fitness is given by the

641 amount and distribution of resources available for its establishment and adaptation (Shelford, 1931).

642 Thus, we reinterpret this concept by assuming that the relation between input availability (deficit,

643 optimum amount, or excess) across different crop development stages drives the amount of the genetic

644 potential expressed in phenotypes produced by the same genotype for a given environment. Therefore,

645 it provides the foundations to elaborate the argument that there is also an indissoluble envirotype- 
646 phenotype covariance in the phenotypic records that is interpreted as a $\mathrm{G} \times \mathrm{E}$ interaction for each

647 environment. Because of that, we envisage that any environmental relatedness kernel must account for

648 it in any way.

649 The pioneer approaches to measuring crop adaptability use the average value of a given trait in

650 a given environment as an environmental quality index (e.g., Finlay and Wilkinson, 1963). However,

651 the problem with this approach is that it explains the quality of the environment realized by the

652 genotypes evaluated in it, making it inefficient to explain the drivers of environmental quality and 653 incapable of predicting untested growing conditions, as observed in our results for Case 2 using 654 GBLUP without enviromic data. In addition, our results for Case 1 highlight that it is a limit in accuracy 655 for traditional GBLUP across MET, in which the accuracy remains almost the same, regardless of the 656 number of phenotypic records available.

A second intrinsic covariance can interpret this last result within the phenotypic records, which 658 is the genotype-envirotype covariance. By adapting the Quantitative Genetics theory to the terminology 659 used here, we can infer that each genotype reacts differently to each envirotype, resulting in a given 660 phenotype. This phenotype is then used to provide small crop phenology differences (genetically 661 determined window sizes for each development stage). Recent but pioneer works have been carried 662 out to understand the genetic and environmental determinants of flowering time in sorghum (Li et al., 663 2018) and rice (Guo et al., 2020). That can be indirectly interpreted as cardinal differential thresholds

664 for temperature response. Jarquín et al (2020) proved that it is possible to increase the ability of GP in 665 predictive novel G×E by coupling information of day-length in the benchmark GP models. For all these 666 examples reported above, we can infer that, when trying to predict a novel genotype, by borrowing 667 genotypic information from the relatives at different environments, it is impossible to reproduce the 668 genotype-envirotype covariance without adding any enviromic information into the model. 
670 the gains in the predictive ability due to the use of multi-environment GP models in contrast to singleenvironment GP models (Bandeira e Souza et al., 2017; de Oliveira et al., 2020) and why deep learning approaches have successfully captured intrinsic $\mathrm{G} \times \mathrm{E}$ patterns and translated them into gains in

673 accuracy (Montesinos-López et al., 2018; Crossa et al., 2019; Cuevas et al., 2019). Conversely, this

674 also might explain the need to incorporate secondary sources of information in the prediction of grain

675 yields across multiple environments (Westhues et al., 2017; Ly et al., 2018; Millet et al., 2019; Costa-

676 Neto et al., 2021a; 2021b; Jarquín et al., 2020), as well as the possible limitations of CGM approaches

677 contrasting scenarios differing from those targeted near-iso conditions of CGM calibration (e.g.,

678 Cooper et al., 2016; Messina et al., 2018). Thus, an alternative can be supervised approaches to

679 describe the environmental relatedness, such as in this paper, and perhaps unsupervised algorithms

680 capable of taking advantage of the covariances related to the genotype-phenotype, genotype-

681 envirotype, and envirotype-phenotype dynamics.

\subsection{Sometimes main-effect enviromics is better than reaction-norm models}

Our results from Case 1 show that the inclusion of enviromic sources (for main-effects or

explicitly incorporated in the $\mathrm{RN}-\mathrm{G} \times \mathrm{E}$ structure) led to a better description of the envirotype-phenotype

highlighting that incorporating enviromic sources does not replace the incorporation of a design matrix

687 for environments (here used as fixed effects) as it is commonly associated in previous studies of GP

688 reaction-norm. Here we show that enviromic sources came up as tentative to capture the envirotype-

689 phenotype covariances. The cross-validation scheme used in Case 1 allowed us to observe that the joint

690 prediction of different genotype-environment conditions (Fig 3) might better highlight how enviromic

691 sources can contribute to increasing the predictive ability of GP, mostly due to its usefulness in

692 approaching the environmental correlation among field trials. It shows more transparency for the 
693 influence of the scenarios $\mathrm{G}, n \mathrm{E}$ and $n \mathrm{G} n \mathrm{E}$, in which we had a considerable lack of phenotypic

694 information in training GP. We can infer that schemes such as CV1 (only $n \mathrm{G}, \mathrm{E}$ ) are the least adequate

695 option to show the benefits of coupling enviromics in GBLUP. However, looking at a drastically sparse

696 MET condition (joint prediction scenarios) shows that enviromics improves the accuracy of GP as the

697 size of the MET also increases. Predictions are made up of tiny experimental networks.

\subsection{Differences in using environmental covariables $(W)$ and typologies $(T)$}

Regarding the enviromic assembly approaches used in this study, there was evidence that using

typologies as envirotype descriptors ( $\mathbf{T}$ matrix) is more biologically accurate in representing

environmental relatedness than quantitative descriptors ( $\mathbf{W}$ matrix) based on quantile covariables. This

increase in biological accuracy was reflected in the statistical accuracy and then boosted plant breeders'

ability to carry out selections across multi-environment conditions. Further efforts in this sense must

be devoted to increasing the level of explanation of the genotype-envirotype covariances, which can

also take advantage of Shelford's Law to refine the limits of tolerance for particular genotypes. Thus,

different genotypes will be under the influence of a diverse set of envirotypes, which can be realized

for the same environmental factor (e.g., solar radiation, air temperature, soil moisture) according to its

occurrence across crop lifetime (e.g., vegetative stage) and the adaptation zone designed from

ecophysiology concepts (e.g., temperature cardinals defining which temperature level results in stress and optimum growing condition).

A second difference may be explained by the fact that quantitative environmental covariates are

713 (2020), and we adapted the idea of envirotypes as markers of environment relatedness in a different

714 manner. For example, the common use of mean values of covariates such as rainfall, solar radiation,

715 and air temperature, in reality, represents a non-additive between each other; yet, they are very well 
716

717

718

719

720

721

722

723

724

725

726

727

728

730

731

732

733

734

735

736

737

738

739

correlated for a given site-planting date condition, even when using strategies to deal with collinearity, such as partial least squares (e.g., Vargas et al., 2006; Porker et al., 2020;). We can use an example as a given day of crop growing in which a large amount of rainfall has occurred. We can suppose that the sky is cloudy, with less radiation and lower temperature. Thus, using such G-BLUP inspired approach is not an ideal solution to estimate the environmental variance. Conversely, the environmental typologies (T) are based on frequencies (ranging from 0 to 1), where the sum of all frequencies are equal to 1 (100\% of the variation). In addition, those typologies can be built for a given site using historical weather data, adapting the approach of Gillberg et al. (2019) and de los Campos et al. (2020). As presented in section 2.4.2, if no typologies are considered, the expected environment effect is given for a fixed-environment intercept (with 0 variance within and between environments). Despite this fact, another option is using nonlinear kernel methods to estimate only the environment-relatedness, as this approach takes advantage of nonlinear relationships among covariates (Costa-Neto et al., 2021a,b).

\subsection{Does more phenotype data mean more accuracy in multi-environment prediction?}

This study shows that environmental information can break the paradigm that claims that more phenotype information leads to greater accuracy of GP models over MET. Our results highlight that the traditional GBLUP models assume that the variation due to $\mathrm{G} \times \mathrm{E}$ is purely genomic-based across field trials, leading to an implicit conclusion that the yield plasticity is constant (slope $\sim 0$ ) for all genotypes, which is unrealistic. It also reflects that $\mathrm{G} \times \mathrm{E}$ patterns are non-crossover (scale changes in performance across different variations), that is, a well-performing genotype will always be good across environments, and a poorly performing genotype has the same trend for all environments. Despite the gains achieved in predicting the quality of a novel environment and the plasticity for tested and untested genotypes, we noticed that the inclusion of enviromic sources also leads to the unrealistic conclusion that all genotypes respond in the same way the gradient of climate and soil quality. Our results show a reasonable accuracy in predicting yield plasticity, but further efforts must be made to 
740 improve this approach's explanation of the yield plasticity as a nonlinear variation across the gradient

741 of environmental factors.

742 The use of selective phenotyping strategies made up with enviromic assembly $\times$ genomic

743 kinships showed a drastic reduction of in-field efforts. Combined with enviromic-aided GBLUP

744 models, it led to almost the same predictive ability achieved using a wide number of genotypes and

745 environments for a large experimental network. Thus, we can enumerate the benefits of the enviromic

746 approaches tested in this study as (1) the possibility of training prediction models for yield plasticity

747 with reduced phenotyping efforts, (2) a consequence of the assembly of enviromics with genomics

748 allowing the selection of the genotype-environment combinations that best represents the main inner

749 covariances among phenotypes produced by different environments (the genotype-phenotype,

750 envirotype-phenotype dynamics mentioned above).

Considering both enviromics approached, we conclude that the advantages of E-GP over W-GP

can be enumerated as (1) the flexibility to design a wide number of environment-types assuming of historical weather and in-field records to compute trends of certain envirotypes at certain environments, which can be coupled into (3) the definition of TPE and characterization of megaenvironments, as the main approach used for this relies on the study of the frequency of occurrence of

757 the main environment-types (e.g., Heinemann et al., 2019). For the latter, for example, the T matrix proposed here is just an arrangement of an environment $\times$ typology matrix, in which each entry represents its frequency of occurrence at a particular time interval of the crop lifetime. Conversely, the

760 advantages of W-GP over E-GP rely on plasticity in creating large-scale envirotype descriptors with 761 reasonable biological accuracy. 


\subsection{Can we envisage climate-smart solutions from enviromics with genomics?}

Modern plant breeding programs must deliver climate-smart solutions cost-effectively and time-

765

766

767

768

769

770

771

772

773

774

775

776

777

778

779

780

781

782

783

784

785

786

reduced (Crossa et al., 2021). By climate-smart solutions, we mean (1) adopting cost-effective approaches capable of providing fast and cheap solutions to face climate change (2) a better resource allocation for field trial efforts to collect representative phenotype information to feed prediction-based platforms for crop improvement, such as training accurate GP models and CGM-based approaches capable of guiding several breeding decisions, (3) a better understanding of which envirotypes most limit the adaptation of crops across the breeding TPE, revising historical trends and expecting future scenarios (e.g., Ramirez-Villegas et al., 2018; 2020; Heinemann et al., 2019) (4) understanding the relationship between secondary traits and their importance in explaining the plant-environment dynamics for given germplasm at given TPE (e.g., Cooper et al., 2021). However, most of those objectives will be hampered if the MET-GP platforms do not consider models with a higher biological meaning (Hammer et al., 2019) and reliable environmental information. A cost-effective solution for that, if the breeder has no access to sensor network tools, relies on the use of remote sensing tools to collect and process basic weather and soil data, such as those available in the EnvRtype R package (Costa-Neto et al., 2021b).

If selective phenotyping is added in the enviromics-aided pipeline for GP (Supplementary Fig 1), additional traits and the possibility of screening genotypes across a wide number of managed environments will increase. It can support field trials' training for CGM approaches, which demands phenotyping of traits across crop life, such as biomass accumulation and partitioning among different plant organs. Finally, using models considering an explicit environmental gradient of keyenvironmental factors is a second alternative for this approach. It can be done to discover the genetic determinants of the interplay between plant plasticity and environment variation. As a wide range of genes reacts to each gradient of environmental factors, the use of whole-genome regressions of 
reaction-norm for each environmental factor must be useful to screen potential genotypes (in our case, single-crosses) for a diverse set of scenarios (e.g., increased heat stress). Pioneer works used this methodology in wheat breeding (Heslot et al., 2014; Ly et al., 2018) inspired other cereal crop applications.

For example, Millet et al. (2019) fine-tuned the methodology by creating a two-stage analysis of al., 2019) and intercept radiation at the vegetative phase (Millet et al., 2019) are the main drivers of to use genotypic-specific regressions, which coupled with genomic data, led to an increase of the accuracy of MET-GP by 55\% concerning the benchmark environmental similarity model made up of mean values of environmental factors, as proposed by Jarquín et al. (2014).

From the aspects mentioned above, we envisage that the use of GP for multi-environment

802 predictions must account for some degree of ecophysiological reality while also considering the 803 balance and the relation between parsimony and accuracy (Hammer et al., 2019; Costa-Neto et al., 804 2021b; Cooper et al., 2021). Here we also highlight in our literature review that multi-environment GP 805 must account for the impact of (1) resource availability in the creation of biologically accurate 806 platforms in training CGM-based approaches and delivering reliable envirotyping information for 807 those purposes, (2) availability of the knowledge of experts in training CGM approaches. Thus, 808 ecophysiology concepts to provide solutions for raw environmental data processing in enviromic 809 assembly information for predictive purposes seem to be a cost-effective alternative to leverage 810 accuracy involving parsimony and biological reality. 


\section{REFERENCES}

812 Allard, R. W., and Bradshaw, A. D. (1964). Implications of genotype-environmental interactions in applied 813 plant breeding. Crop Sci. 4, 503-508.

814 Allen, R. G., Pereira, L. S., Raes, D., and Smith, M. (1998). Crop Evapotranspiration - guidelines for computing crop water requirements. 56th ed. Rome: FAO Irrigation and Drainage Paper $\mathrm{N}^{\circ} 56$.

Antolin, L. A. S., and Heinemann, A. B. (2021). Impact assessment of common bean availability in Brazil under climate change scenarios. 191. doi:10.1016/j.agsy.2021.103174.

Arnold, P. A., Kruuk, L. E. B., and Nicotra, A. B. (2019). How to analyse plant phenotypic plasticity in response to a changing climate. New Phytol. 222, 1235-1241. doi:10.1111/nph.15656.

Alves, F. C., Granato, Í. S. C., Galli, G., Lyra, D. H., Fritsche-Neto, R., and De Los Campos, G. (2019). Bayesian analysis and prediction of hybrid performance. Plant Methods 15, 1-18. doi:10.1186/s13007019-0388-x.

Akdemir, D., and Isidro-Sánchez, J. (2019). Design of training populations for selective phenotyping in genomic prediction. Sci. Rep. 9, 1-15. doi:10.1038/s41598-018-38081-6.

Bandeira e Souza, M., Cuevas, J., Couto, E. G. de O., Pérez-Rodríguez, P., Jarquín, D., Fritsche-Neto, R., et al. (2017). Genomic-Enabled Prediction in Maize Using Kernel Models with Genotype $\times$ Environment Interaction. G3 7, g3.117.042341. doi:10.1534/g3.117.042341.

Bournan, B. A. M., Keulen, H. Van, and Rabbingeh, R. (1996). The 'School of de Wit' Crop Growth Simulation Models : A Pedigree and Historical Overview. 52.

830 Bradshaw, A. D. (1965). Evolutionary significance of phenotypic plasticity in plants. Adv. Genet. 13, 115-

$831 \quad 155$.

Cimen, E., Jensen, S. E., and Buckler, E. S. (2021). Building a tRNA thermometer to estimate microbial adaptation to temperature. Nucleic Acids Res. 48, 12004-12015. doi:10.1093/nar/gkaa1030.

Cooper, M., Messina, C. D., Podlich, D., Totir, L. R., Baumgarten, A., Hausmann, N. J., et al. (2014). Predicting the future of plant breeding: Complementing empirical evaluation with genetic prediction. Crop Pasture Sci. 65, 311-336. doi:10.1071/CP14007.

Cooper, M., Technow, F., Messina, C., Gho, C., and Radu Totir, L. (2016). Use of crop growth models with whole-genome prediction: Application to a maize multienvironment trial. Crop Sci. 56, 2141-2156. doi:10.2135/cropsci2015.08.0512.

840 Cooper, M., Powell, O., Voss-Fels, K. P., Messina, C. D., Gho, C., Podlich, D. W., et al. (2021). Modelling selection response in plant-breeding programs using crop models as mechanistic gene-to-phenotype (CGM-G2P) multi-trait link functions. in silico Plants 3, 1-21. doi:10.1093/insilicoplants/diaa016.

843 Cortés, A. J., Restrepo-Montoya, M., and Bedoya-Canas, L. E. (2020). Modern Strategies to Assess and Breed 
Costa-Neto, G., Fritsche-Neto, R., and Crossa, J. (2021a). Nonlinear kernels, dominance, and envirotyping data increase the accuracy of genome-based prediction in multi-environment trials. Heredity (Edinb). 126, 92-106. doi:10.1038/s41437-020-00353-1.

Costa-Neto, G., Galli, G., Carvalho, H. F., Crossa, J., and Fritsche-Neto, R. (2021b). EnvRtype: a software to interplay enviromics and quantitative genomics in agriculture. G3 Genes|Genomes|Genetics.

doi:10.1093/g3journal/jkab040.

Crossa, J., Pérez-Rodríguez, P., Cuevas, J., Montesinos-López, O., Jarquín, D., de los Campos, G., et al. (2017). Genomic Selection in Plant Breeding: Methods, Models, and Perspectives. Trends Plant Sci. 22, 961-975. doi:10.1016/j.tplants.2017.08.011.

Crossa, J., Martini, J. W. R., Gianola, D., Pérez-Rodríguez, P., Jarquin, D., Juliana, P., et al. (2019). Deep Kernel and Deep Learning for Genome-Based Prediction of Single Traits in Multienvironment Breeding Trials. Front. Genet. 10, 1-13. doi:10.3389/fgene.2019.01168

Crossa, J., Fritsche-Neto, R., Montesinos-lopez, O. A., Costa-Neto, G., Dreisigacker, S., Montesinos-lopez, A., et al. (2021). The Modern Plant Breeding Triangle: Optimizing the Use of Genomics, Phenomics, and Enviromics Data. Front. Plant Sci. 12, 1-6. doi:10.3389/fpls.2021.651480.

Cuevas, J., Montesinos-López, O., Juliana, P., Guzmán, C., Pérez-Rodríguez, P., González-Bucio, J., et al. (2019). Deep Kernel for genomic and near infrared predictions in multi-environment breeding trials. G3 Genes, Genomes, Genet. 9, 2913-2924. doi:10.1534/g3.119.400493.

de los Campos, G., Pérez-Rodríguez, P., Bogard, M., Gouache, D., and Crossa, J. (2020). A data-driven simulation platform to predict cultivars' performances under uncertain weather conditions. Nat. Commun. 11. doi:10.1038/s41467-020-18480-y.

de Oliveira, A. A., Resende, M. F. R., Ferrão, L. F. V., Amadeu, R. R., Guimarães, L. J. M., Guimarães, C. T., et al. (2020). Genomic prediction applied to multiple traits and environments in second season maize hybrids. Heredity (Edinb). 125, 60-72. doi:10.1038/s41437-020-0321-0.

Dias, K. O. D. G., Gezan, S. A., Guimarães, C. T., Nazarian, A., Da Costa E Silva, L., Parentoni, S. N., et al. (2018). Improving accuracies of genomic predictions for drought tolerance in maize by joint modeling of additive and dominance effects in multi-environment trials. Heredity (Edinb). 121, 24-37. doi:10.1038/s41437-018-0053-6.

Epinat-Le Signor, C., Dousse, S., Lorgeou, J., Denis, J.-B., Bonhomme, R., Carolo, P., et al. (2001). Interpretation of genotype x environment interactions for early maize hybrids over 12 years. Crop Sci. 41, 663-669. doi:10.2135/cropsci2001.413663x.

Finlay, K. W., and Wilkinson, G. N. (1963). The analysis of adaptation in a plant breeding programme. $J$. Agric. Res. 14, 742-754.

Gage, J. L., Jarquin, D., Romay, C., Lorenz, A., Buckler, E. S., Kaeppler, S., et al. (2017). The effect of artificial selection on phenotypic plasticity in maize. Nat. Commun. 8. doi:10.1038/s41467-017-01450-2.

Galli, G., Sabadin, F., Costa-Neto, G. M. F., and Fritsche-Neto, R. (2021). A novel way to validate UAS-based high-throughput phenotyping protocols using in silico experiments for plant breeding purposes. Theor. Appl. Genet. 134, 715-730. doi:10.1007/s00122-020-03726-6. 
883

884

885

886

887

888

889

890

891

892

893

894

895

896

897

898

899

900

901

902

903

904

905

906

907

908

909

910

911

912

913

914

915

916

917

918

919

920

Gillberg, J., Marttinen, P., Mamitsuka, H., and Kaski, S. (2019). Modelling G×E with historical weather information improves genomic prediction in new environments. Bioinformatics 35, 4045-4052. doi:10.1093/bioinformatics/btz197.

Granato, I., Cuevas, J., Luna-Vázquez, F., Crossa, J., Montesinos-López, O., Burgueño, J., et al. (2018). BGGE: A new package for genomic-enabled prediction incorporating genotype $\times$ environment interaction models. G3 Genes, Genomes, Genet. 8, 3039-3047. doi:10.1534/g3.118.200435.

Guo, T., Mu, Q., Wang, J., Vanous, A. E., Onogi, A., Iwata, H., et al. (2020). Dynamic effects of interacting genes underlying rice flowering-time phenotypic plasticity and global adaptation. Genome Res. 30, 673683. doi:10.1101/gr.255703.119.

Hammer, G., Messina, C., Wu, A., and Cooper, M. (2019). Biological reality and parsimony in crop models why we need both in crop improvement! 1-21. doi:10.1093/insilicoplants/diz010.

Heinemann, A. B., Ramirez-Villegas, J., Rebolledo, M. C., Costa Neto, G. M. F., and Castro, A. P. (2019). Upland rice breeding led to increased drought sensitivity in Brazil. F. Crop. Res. 231, 57-67. doi:10.1016/j.fcr.2018.11.009.

Heslot, N., Akdemir, D., Sorrells, M. E., and Jannink, J.-L. (2014). Integrating environmental covariates and crop modeling into the genomic selection framework to predict genotype by environment interactions. Theor. Appl. Genet. 127, 463-480.

Jarquín, D., Crossa, J., Lacaze, X., Du Cheyron, P., Daucourt, J., Lorgeou, J., et al. (2014). A reaction norm model for genomic selection using high-dimensional genomic and environmental data. Theor. Appl. Genet. 127, 595-607. doi:10.1007/s00122-013-2243-1.

Jarquin, D., Kajiya-Kanegae, H., Taishen, C., Yabe, S., Persa, R., Yu, J., et al. (2020). Coupling day length data and genomic prediction tools for predicting time-related traits under complex scenarios. Sci. Rep. 10, 1-12. doi:10.1038/s41598-020-70267-9.

Jończyk, M., Sobkowiak, A., Trzcinska-Danielewicz, J., Skoneczny, M., Solecka, D., Fronk, J., et al. (2017). Global analysis of gene expression in maize leaves treated with low temperature. II. Combined effect of severe cold $\left(8^{\circ} \mathrm{C}\right)$ and circadian rhythm. Plant Mol. Biol. 95, 279-302. doi:10.1007/s11103-017-0651-3.

Lorenzana, R. E., and Bernardo, R. (2009). Accuracy of genotypic value predictions for marker-based selection in biparental plant populations. Theor. Appl. Genet. 120, 151-161. doi:10.1007/s00122-0091166-3.

Li, X., Guo, T., Mu, Q., Li, X., and Yu, J. (2018). Genomic and environmental determinants and their interplay underlying phenotypic plasticity. Proc. Natl. Acad. Sci. U. S. A. 115, 6679-6684. doi:10.1073/pnas.1718326115.

Liu, S., Li, C., Wang, H., Wang, S., Yang, S., Liu, X., et al. (2020). Mapping regulatory variants controlling gene expression in drought response and tolerance in maize. Genome Biol. 21, 1-22. doi:10.1186/s13059-020-02069-1.

Ly, D., Huet, S., Gauffreteau, A., Rincent, R., Touzy, G., Mini, A., et al. (2018). Whole-genome prediction of reaction norms to environmental stress in bread wheat ( Triticum aestivum L.) by genomic random regression. F. Crop. Res. 216, 32-41. doi:10.1016/j.fcr.2017.08.020. 
932

933

934

935

936

937

938

939

940

941

942

943

944

945

946

947

948

949

950

951

952

953

954

955

956

957

958

Martini, J. W. R., Crossa, J., Toledo, F. H., and Cuevas, J. (2020). On Hadamard and Kronecker products in covariance structures for genotype $\times$ environment interaction. Plant Genome 13, 1-12. doi:10.1002/tpg2.20033.

Messina, C. D., Technow, F., Tang, T., Totir, R., Gho, C., and Cooper, M. (2018). Leveraging biological insight and environmental variation to improve phenotypic prediction: Integrating crop growth models (CGM) with whole genome prediction (WGP). Eur. J. Agron. 100, 151-162.

Meuwissen, T. H. E., Hayes, B. J., and Goddard, M. E. (2001). Prediction of total genetic value using genomewide dense marker maps. Genetics 157, 1819-1829.

Montesinos-López, A., Montesinos-López, O. A., Gianola, D., Crossa, J., and Hernández-Suárez, C. M. (2018). Multi-environment genomic prediction of plant traits using deep learners with dense architecture. G3 Genes, Genomes, Genet. 8, 3813-3828. doi:10.1534/g3.118.200740.

Millet, E. J., Kruijer, W., Coupel-Ledru, A., Alvarez Prado, S., Cabrera-Bosquet, L., Lacube, S., et al. (2019). Genomic prediction of maize yield across European environmental conditions. Nat. Genet. 51, pages952-956. doi:10.1038/s41588-019-0414-y.

Misztal, I. (2016). Inexpensive computation of the inverse of the genomic relationship matrix in populations with small effective population size. Genetics 202, 401-409. doi:10.1534/genetics.115.182089.

Morais Júnior, O. P., Duarte, J. B., Breseghello, F., Coelho, A. S. G., and Magalhães Júnior, A. M. (2018). Single-step reaction norm models for genomic prediction in multienvironment recurrent selection trials. Crop Sci. 58, 592-607. doi:10.2135/cropsci2017.06.0366.

Porker, K., Coventry, S., Fettell, N. A., Cozzolino, D., and Eglinton, J. (2020). Using a novel PLS approach for envirotyping of barley phenology and adaptation. F. Crop. Res. 246, 1-11. doi:10.1016/j.fcr.2019.107697.

Ramirez-Villegas, J., Heinemann, A. B., Castro, A. P., Breseghello, F., Navarro-Racines, C., Li, T., et al. (2018). Breeding implications of drought stress under future climate for upland rice in Brazil. Glob. Chang. Biol. 1, 1-16. doi:10.1111/ijlh.12426.

Ramirez-Villegas, J., Molero Milan, A., Alexandrov, N., Asseng, S., Challinor, A. J., Crossa, J., et al. (2020). CGIAR modeling approaches for resource-constrained scenarios: I. Accelerating crop breeding for a changing climate. Crop Sci. 60, 547-567. doi:10.1002/csc2.20048.

Resende, R. T., Piepho, H. P., Rosa, G. J. M., Silva-Junior, O. B., e Silva, F. F., de Resende, M. D. V., et al. (2020). Enviromics in breeding: applications and perspectives on envirotypic-assisted selection. Theor. Appl. Genet. doi:10.1007/s00122-020-03684-z.

Robert, P., Le Gouis, J., and Rincent, R. (2020). Combining Crop Growth Modeling With Trait-Assisted Prediction Improved the Prediction of Genotype by Environment Interactions. Front. Plant Sci. 11, 1-11. doi:10.3389/fpls.2020.00827.

Rogers, A. R., Dunne, J. C., Romay, C., Bohn, M., Buckler, E. S., Ciampitti, I. A., et al. (2021). The importance of dominance and genotype-by-environment interactions on grain yield variation in a largescale public cooperative maize experiment. G3 Genes|Genomes|Genetics 11. doi:10.1093/g3journal/jkaa050. 
959

960

961

962

963

964

965

966

967

968

969

970

971

972

973

974

975

976

977

978

979

980

981

982

983

984

985

986

987

988

989

990

991

992

993

994

995

996

Romay, M. C., Malvar, R. A., Campo, L., Alvarez, A., Moreno-González, J., Ordás, A., et al. (2010). Climatic and genotypic effects for grain yield in maize under stress conditions. Crop Sci. 50, 51-58.

Shelford, V. . E. . (1931). Some Concepts of Bioecology. Ecology 12, 455-467.

Sparks, A. (2018). nasapower: A NASA POWER Global Meteorology, Surface Solar Energy and Climatology Data Client for R. J. Open Source Softw. 3, 1035. doi:10.21105/joss.01035.

Tigchelaar, M., Battisti, D. S., Naylor, R. L., and Ray, D. K. (2018). Future warming increases probability of globally synchronized maize production shocks. Proc. Natl. Acad. Sci. U. S. A. 115, 6644-6649. doi:10.1073/pnas.1718031115.

Toda, Y., Wakatsuki, H., Aoike, T., Kajiya-Kanegae, H., Yamasaki, M., Yoshioka, T., et al. (2020). Predicting biomass of rice with intermediate traits: Modeling method combining crop growth models and genomic prediction models. PLoS One 15, 1-21. doi:10.1371/journal.pone.0233951.

Unterseer, S., Bauer, E., Haberer, G., Seidel, M., Knaak, C., Ouzunova, M., et al. (2014). A powerful tool for genome analysis in maize: Development and evaluation of the high density $600 \mathrm{k}$ SNP genotyping array. BMC Genomics 15, 1-15. doi:10.1186/1471-2164-15-823.

Vargas, M., Van Eeuwijk, F. A., Crossa, J., and Ribaut, J. M. (2006). Mapping QTLs and QTL x environment interaction for CIMMYT maize drought stress program using factorial regression and partial least squares methods. Theor. Appl. Genet. 112, 1009-1023. doi:10.1007/s00122-005-0204-z.

Voss-Fels, K. P., Cooper, M., and Hayes, B. J. (2019). Accelerating crop genetic gains with genomic selection. Theor. Appl. Genet. 132, 669-686. doi:10.1007/s00122-018-3270-8.

Vendramin, S., Huang, J., Crisp, P. A., Madzima, T. F., and McGinnis, K. M. (2020). Epigenetic regulation of ABA-induced transcriptional responses in maize. G3 Genes, Genomes, Genet. 10, 1727-1743. doi:10.1534/g3.119.400993.

Vidotti, M. S., Matias, F. I., Alves, F. C., Rodríguez, P. P., Beltran, G. A., Burguenõ, J., et al. (2019). Maize responsiveness to Azospirillum brasilense: Insights into genetic control, heterosis and genomic prediction. PLoS One 14, 1-22. doi:10.1371/journal.pone.0217571.

Westhues, M., Schrag, T. A., Heuer, C., Thaller, G., Utz, H. F., Schipprack, W., et al. (2017). Omics-based hybrid prediction in maize. Theor. Appl. Genet. doi:10.1007/s00122-017-2934-0.

Windhausen, V. S., Atlin, G. N., Hickey, J. M., Crossa, J., Jannink, J.-L., Sorrells, M. E., et al. (2012). Effectiveness of Genomic Prediction of Maize Hybrid Performance in Different Breeding Populations and Environments. G3:Genes|Genomes|Genetics 2, 1427-1436. doi:10.1534/g3.112.003699.

$\mathrm{Xu}, \mathrm{Y}$. (2016). Envirotyping for deciphering environmental impacts on crop plants. Theor. Appl. Genet. 129, 653-673. doi:10.1007/s00122-016-2691-5.

Zhao, Y., Gowda, M., Liu, W., Würschum, T., Maurer, H. P., Longin, F. H., et al. (2012). Accuracy of genomic selection in European maize elite breeding populations. Theor. Appl. Genet. 124, 769-776. doi:10.1007/s00122-011-1745-y.

Zhang, X., Pérez-Rodríguez, P., Semagn, K., Beyene, Y., Babu, R., López-Cruz, M. A., et al. (2015). Genomic prediction in biparental tropical maize populations in water-stressed and well-watered environments using low-density and GBS SNPs. Heredity (Edinb). 114, 291-299. doi:10.1038/hdy.2014.99. 


\section{TABLES}

998 Table 1. Predictive ability ( \pm standard error) of the genome-based prediction models (GP) for the N-level set of 999 tropical maize hybrids ( 570 hybrids $\times 2$ locations $\times$ two years $\times$ two nitrogen managements). Bold values denote 1000 higher predictive ability values for each scenario: G,E (known genotypes at known growing conditions), G, $n \mathrm{E}$ 1001 (known genotypes at new growing conditions), $n \mathrm{G}, \mathrm{E}$ (new genotypes at known growing conditions), and $n \mathrm{G}$, $1002 n \mathrm{E}$ (new genotypes at new growing conditions).

\begin{tabular}{cccccc}
\hline \multirow{2}{*}{ Training Setup } & \multirow{2}{*}{ Model } & \multicolumn{4}{c}{ Prediction Scenario } \\
\cline { 3 - 6 } & GBLUP & $0.771 \pm 0.064$ & $0.397 \pm 0.046$ & $0.310 \pm 0.054$ & $0.297 \pm 0.029$ \\
& E-GP (BD) & $\mathbf{0 . 9 0 3} \pm 0.115$ & $\mathbf{0 . 4 9 3} \pm 0.169$ & $\mathbf{0 . 6 1 5} \pm 0.022$ & $\mathbf{0 . 4 1 6} \pm 0.153$ \\
$7 / 8$ & E-GP (RN) & $0.833 \pm 0.118$ & $\mathbf{0 . 4 7 7} \pm 0.199$ & $\mathbf{0 . 6 1 3} \pm 0.040$ & $0.394 \pm 0.193$ \\
Environments & W-GP (BD) & $\mathbf{0 . 9 1 5} \pm 0.115$ & $0.333 \pm 0.208$ & $\mathbf{0 . 6 1 4} \pm 0.025$ & $0.242 \pm 0.189$ \\
& W-GP (RN) & $0.885 \pm 0.117$ & $0.327 \pm 0.210$ & $0.613 \pm 0.031$ & $0.23 \pm 0.196$ \\
\cline { 2 - 6 } & GBLUP & $0.747 \pm 0.049$ & $0.432 \pm 0.046$ & $0.294 \pm 0.026$ & $0.323 \pm 0.04$ \\
& E-GP (BD) & $0.905 \pm 0.056$ & $\mathbf{0 . 5 5 4} \pm 0.144$ & $\mathbf{0 . 6 5 9} \pm 0.015$ & $\mathbf{0 . 4 6 4} \pm 0.113$ \\
$5 / 8$ & E-GP (RN) & $0.833 \pm 0.056$ & $\mathbf{0 . 5 7 0} \pm 0.132$ & $\mathbf{0 . 6 6 0} \pm 0.025$ & $\mathbf{0 . 4 7 5} \pm 0.104$ \\
Environments & W-GP (BD) & $\mathbf{0 . 9 3 1} \pm 0.057$ & $0.449 \pm 0.286$ & $0.659 \pm 0.019$ & $0.347 \pm 0.253$ \\
& W-GP (RN) & $0.897 \pm 0.056$ & $0.501 \pm 0.229$ & $\mathbf{0 . 6 6 0} \pm 0.026$ & $0.395 \pm 0.198$ \\
\cline { 2 - 5 } & GBLUP & $0.739 \pm 0.040$ & $0.527 \pm 0.080$ & $0.295 \pm 0.015$ & $0.394 \pm 0.044$ \\
& E-GP (BD) & $0.899 \pm 0.026$ & $0.534 \pm 0.081$ & $0.660 \pm 0.012$ & $0.388 \pm 0.038$ \\
3/8 & E-GP (RN) & $0.823 \pm 0.026$ & $\mathbf{0 . 5 6 6} \pm 0.086$ & $\mathbf{0 . 6 6 3} \pm 0.015$ & $\mathbf{0 . 4 2 0} \pm 0.041$ \\
Environments & W-GP (BD) & $\mathbf{0 . 9 2 4} \pm 0.026$ & $0.532 \pm 0.08$ & $0.660 \pm 0.015$ & $0.384 \pm 0.038$ \\
& W-GP (RN) & $0.886 \pm 0.025$ & $\mathbf{0 . 5 7 9} \pm 0.088$ & $\mathbf{0 . 6 6 3} \pm 0.020$ & $\mathbf{0 . 4 2 4} \pm 0.041$ \\
\hline
\end{tabular}

1003

1004

1005

1006

1007

1008

1009

1010

Table 2. Predictive ability ( \pm standard error) of the genome-based prediction models (GP) for the Multi-Local set of tropical maize hybrids ( 247 hybrids $\times 5$ locations in different regions of Brazil). Bold values denote the higher predictive ability values for each scenario: G,E (known genotypes at known growing conditions), G, $n \mathrm{E}$ (known genotypes at new growing conditions), $n \mathrm{G}, \mathrm{E}$ (new genotypes at known growing conditions), and $n \mathrm{G}$, $n \mathrm{E}$ (new genotypes at new growing conditions).

\begin{tabular}{cccccc}
\hline \multirow{2}{*}{ Training Setup } & \multirow{2}{*}{ Model } & \multicolumn{4}{c}{ Prediction Scenario } \\
\cline { 2 - 6 } & GBLUP & $0.953 \pm 0.040$ & $0.497 \pm 0.072$ & $0.552 \pm 0.171$ & $0.340 \pm 0.138$ \\
& E-GP (BD) & $\mathbf{0 . 9 8 7} \pm 0.006$ & $\mathbf{0 . 5 2 6} \pm 0.054$ & $\mathbf{0 . 5 9 9} \pm 0.097$ & $\mathbf{0 . 3 6 3} \pm 0.131$ \\
$4 / 5$ & E-GP (RN) & $0.873 \pm 0.084$ & $0.520 \pm 0.064$ & $0.496 \pm 0.126$ & $\mathbf{0 . 3 5 8} \pm 0.143$ \\
Environments & W-GP (BD) & $\mathbf{0 . 9 8 9} \pm 0.005$ & $\mathbf{0 . 5 2 7} \pm 0.056$ & $\mathbf{0 . 5 9 9} \pm 0.098$ & $0.361 \pm 0.131$ \\
& W-GP (RN) & $0.931 \pm 0.057$ & $0.492 \pm 0.078$ & $0.501 \pm 0.130$ & $\mathbf{0 . 3 6 6} \pm 0.125$ \\
\cline { 2 - 6 } & GBLUP & $0.927 \pm 0.045$ & $0.528 \pm 0.066$ & $0.543 \pm 0.208$ & $0.381 \pm 0.142$ \\
& E-GP (BD) & $\mathbf{0 . 9 8 4} \pm 0.006$ & $\mathbf{0 . 5 5 6} \pm 0.052$ & $\mathbf{0 . 5 9 7} \pm 0.097$ & $\mathbf{0 . 4 0 0} \pm 0.131$ \\
$3 / 5$ & E-GP (RN) & $0.845 \pm 0.073$ & $0.550 \pm 0.059$ & $0.477 \pm 0.120$ & $0.385 \pm 0.135$ \\
Environments & W-GP (BD) & $\mathbf{0 . 9 8 7} \pm 0.005$ & $\mathbf{0 . 5 5 5} \pm 0.053$ & $\mathbf{0 . 5 9 8} \pm 0.095$ & $\mathbf{0 . 3 9 4} \pm 0.132$ \\
& W-GP (RN) & $0.915 \pm 0.049$ & $0.514 \pm 0.072$ & $0.483 \pm 0.124$ & $0.392 \pm 0.119$ \\
\cline { 2 - 6 } & GBLUP & $0.913 \pm 0.050$ & $0.552 \pm 0.063$ & $0.538 \pm 0.223$ & $0.409 \pm 0.149$ \\
& E-GP (BD) & $\mathbf{0 . 9 8 2} \pm 0.006$ & $\mathbf{0 . 5 7 4} \pm 0.051$ & $\mathbf{0 . 5 9 3} \pm 0.095$ & $\mathbf{0 . 4 1 0} \pm 0.135$ \\
2/5 & E-GP (RN) & $0.831 \pm 0.069$ & $0.572 \pm 0.060$ & $0.468 \pm 0.117$ & $0.394 \pm 0.134$ \\
Environments & W-GP (BD) & $\mathbf{0 . 9 8 6} \pm .004$ & $\mathbf{0 . 5 7 5} \pm 0.051$ & $\mathbf{0 . 5 9 2} \pm 0.096$ & $\mathbf{0 . 4 1 1} \pm 0.139$ \\
& W-GP (RN) & $0.906 \pm 0.046$ & $0.539 \pm 0.067$ & $0.476 \pm 0.119$ & $0.404 \pm 0.116$ \\
\hline
\end{tabular}

1011 
1013 Table 3. Predictive ability of the genomic prediction models (GP) for two tropical maize data sets (Multi1014 Regional and N-level) produced using the effective number of phenotypic records ( $\mathrm{N}_{\mathrm{GE}}$, genotypes1015 environments observations) and for the scenarios Field Trials (predicting $\mathrm{N}_{\mathrm{GE}}$ ) and Virtual Network (predicting $1016 n-\mathrm{N}_{\mathrm{GE}}$, where $\mathrm{n}$ is the number of genotypes by environments available in the full data set). The reference "full" 1017 and "5\%" in parentheses represents the predictive ability produced with all genotypes and using only the top $10185 \%$, respectively

\begin{tabular}{|c|c|c|c|c|c|}
\hline \multirow{2}{*}{ Scenario } & \multicolumn{5}{|c|}{ Models } \\
\hline & GBLUP & W-GP (BD) & W-GP (RN) & E-GP (BD) & E-GP (RN) \\
\hline \multicolumn{6}{|l|}{$\begin{array}{l}\text { Multi-Regional set } \\
\text { Field Trials }\end{array}$} \\
\hline $\mathrm{N}_{\mathrm{GE}}=210($ full $)$ & 0.698 & 0.962 & 0.892 & 0.964 & 0.893 \\
\hline $\mathrm{N}_{\mathrm{GE}}=210(5 \%)$ & 0.991 & 0.995 & 0.992 & 0.997 & 0.998 \\
\hline $\mathrm{N}_{\mathrm{GE}}=49($ full $)$ & 0.738 & 0.941 & 0.840 & 0.942 & 0.840 \\
\hline $\mathrm{N}_{\mathrm{GE}}=49(5 \%)$ & 0.991 & 0.991 & 0.991 & 1.000 & 1.000 \\
\hline \multicolumn{6}{|l|}{ Virtual Network } \\
\hline $\mathrm{N}_{\mathrm{GE}}=210($ full $)$ & 0.175 & 0.794 & 0.787 & 0.793 & 0.787 \\
\hline $\mathrm{N}_{\mathrm{GE}}=210(5 \%)$ & 0.098 & 0.736 & 0.750 & 0.713 & 0.715 \\
\hline $\mathrm{N}_{\mathrm{GE}}=49($ full $)$ & 0.190 & 0.810 & 0.788 & 0.810 & 0.789 \\
\hline $\mathrm{N}_{\mathrm{GE}}=49(5 \%)$ & 0.241 & 0.759 & 0.755 & 0.758 & 0.706 \\
\hline \multicolumn{6}{|l|}{ N-level set } \\
\hline \multicolumn{6}{|l|}{ Field Trials } \\
\hline $\mathrm{N}_{\mathrm{GE}}=536($ full $)$ & 0.982 & 0.984 & 0.775 & 0.991 & 0.775 \\
\hline $\mathrm{N}_{\mathrm{GE}}=536(5 \%)$ & 0.964 & 0.861 & 0.861 & 0.998 & 0.999 \\
\hline $\mathrm{N}_{\mathrm{GE}}=67($ full $)$ & 0.983 & 0.981 & 0.718 & 0.989 & 0.719 \\
\hline $\mathrm{N}_{\mathrm{GE}}=67(5 \%)$ & 0.967 & 0.833 & 0.802 & 0.998 & 1.000 \\
\hline \multicolumn{6}{|l|}{ Virtual Network } \\
\hline $\mathrm{N}_{\mathrm{GE}}=536($ full $)$ & 0.196 & 0.608 & 0.612 & 0.601 & 0.612 \\
\hline $\mathrm{N}_{\mathrm{GE}}=536(5 \%)$ & 0.152 & 0.554 & 0.545 & 0.406 & 0.484 \\
\hline $\mathrm{N}_{\mathrm{GE}}=67($ full $)$ & 0.102 & 0.574 & 0.572 & 0.578 & 0.573 \\
\hline $\mathrm{N}_{\mathrm{GE}}=67(5 \%)$ & 0.070 & 0.545 & 0.539 & 0.379 & 0.510 \\
\hline
\end{tabular}




\section{Figure Captions}

a

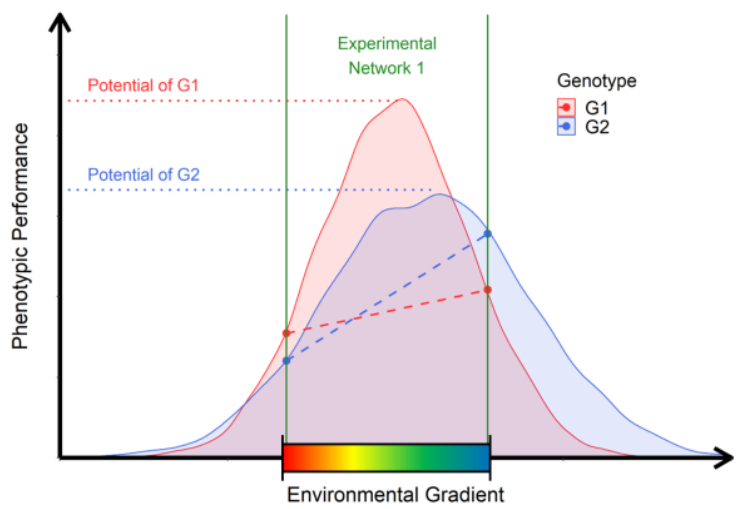

b
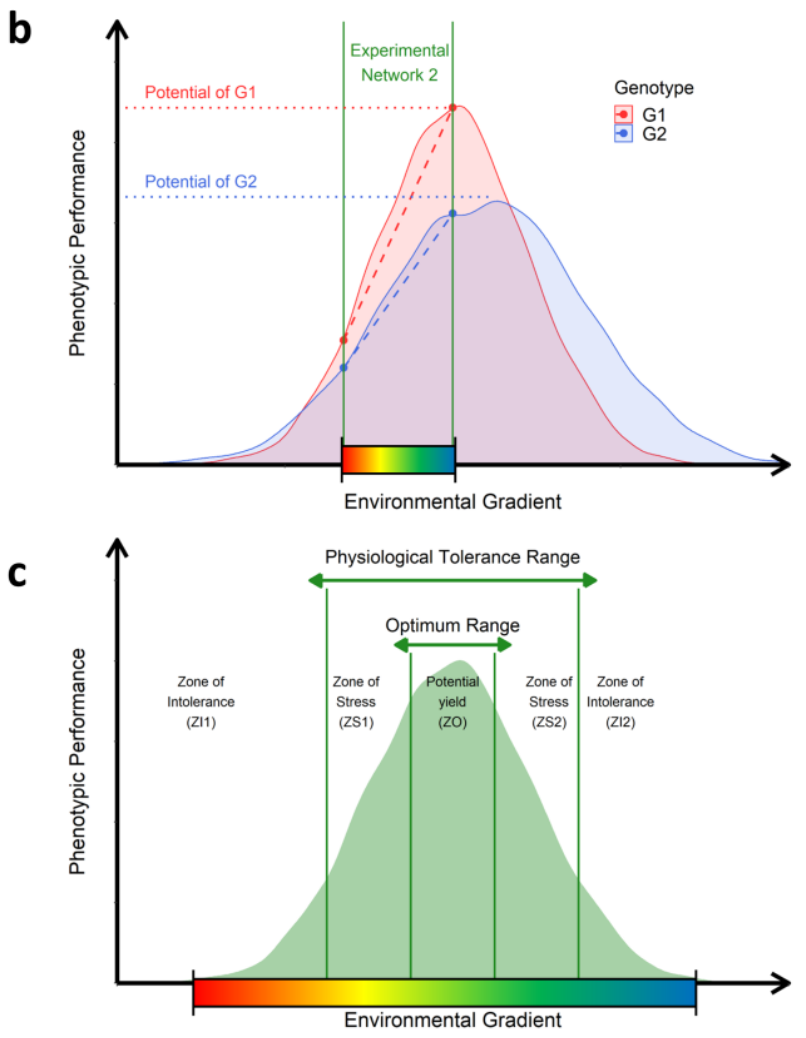

1023 Fig. 1. Ecophysiological insights to translate raw-environmental data into enviromic sources. a. Representation of an experimental network involving an unknown number of environments from a theoretical TPE and two genotypes (G1 and G2). The range of the environmental gradient is delimited by the space between the two vertical green lines. Each genotype has a nonlinear function describing the genetic limits of their phenotypic plasticity (curves) and genetic potential (horizontal dotted lines) of a given trait. Diagonal dotted lines denote the observed reaction-norm experienced by those genotypes; $\mathbf{b}$. representation of a second experimental network involving the same genotypes, but different environments were sampled from the theoretical TPE. c. adaptation of Shelford's Law of Tolerance, describing the cardinal (or biological) genetic limits (vertical green lines) to determine the amount of the factor that results in different adaptation zones. Across these zones, crop performance is described by zones of stress caused by deficit or excess (physiological tolerance range) and zones of optimal growing conditions that allow the plants to express the genetic potential for a given trait (optimum range). The core of possible environmental variations contemplated as putative 
bioRxiv preprint doi: https://doi.org/10.1101/2021.06.04.447091; this version posted June 7, 2021. The copyright holder for this preprint (which was not certified by peer review) is the author/funder. All rights reserved. No reuse allowed without permission.

1035

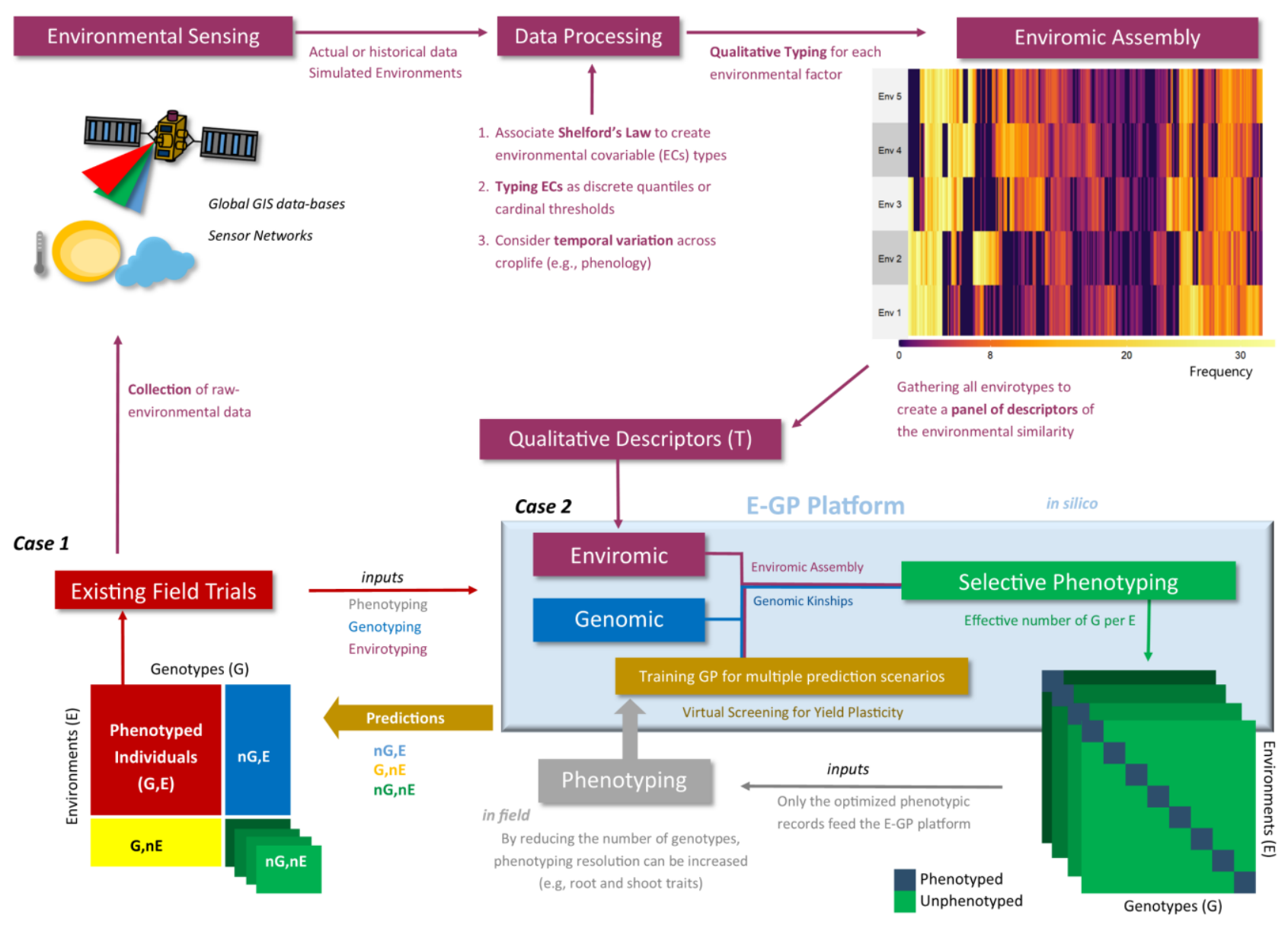

1037 Fig. 2. Workflow of the E-GP considering the two study cases (Case 1 and Case 2) of this study

1038

1039

1040

1041

1042

1043

1044 


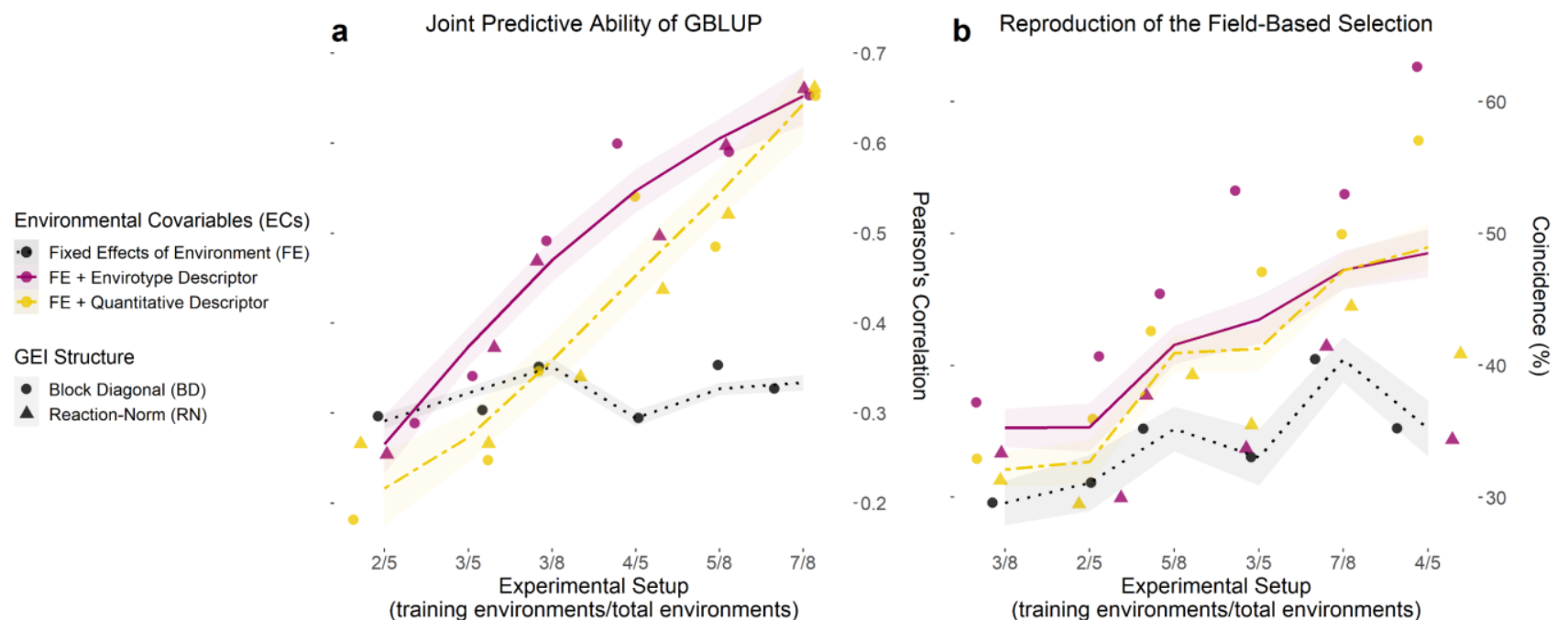

Fig. 3. Joint accuracy trends of GP models for each training setup of existing experimental networks. a. Predictive ability computed with the correlation $(r)$ between observed $(y)$ and predicted $(\hat{y})$ values for the grain yield of each genotype in each environment, over three experimental setups (number of environments used/total of environments) for both maize sets (N-level and Multi-local), using 70\% of the genotypes as a training set and the remaining $30 \%$ as a testing set. b. Coincidence index (CS) between the field-based and prediction-based selection of the best $5 \%$ genotypes in each environment for the same experimental setups and data sets. Dots and triangles represent the point estimates of predictive ability and CS for models involving a block diagonal genomic matrix for G×E effects (dotted) and an enviromic $\times$ genomic reaction-norm G $\times E$ effect (triangle). Trend lines were plotted from the partial values of each sample (from 1 to 50 ) and three prediction scenarios ( $n \mathrm{G}, \mathrm{E} ; \mathrm{G}, n \mathrm{E}$ and $n \mathrm{G}, n \mathrm{E})$ by using the gam() integrated with smoothness estimation in R. Black dotted lines represent the benchmark GBLUP method, considering the effect of the environment as a fixed intercept. Yellow two-dash lines represent the GBLUP involving the main effect from quantitative descriptors (W matrix). Finally, solid dark pink lines represent the GBLUP involving the main effect of envirotype descriptors ( $\mathbf{T}$ matrix). Thus, the latter represents the E-GP based approach for Case 1 (predictions under existing experimental 1059 networks). 
a

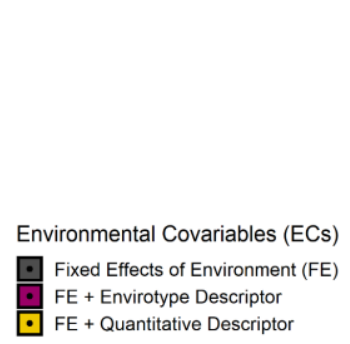

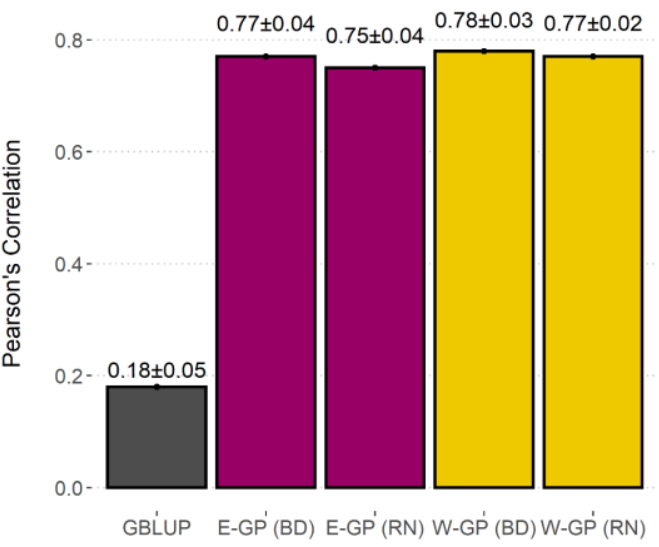

b

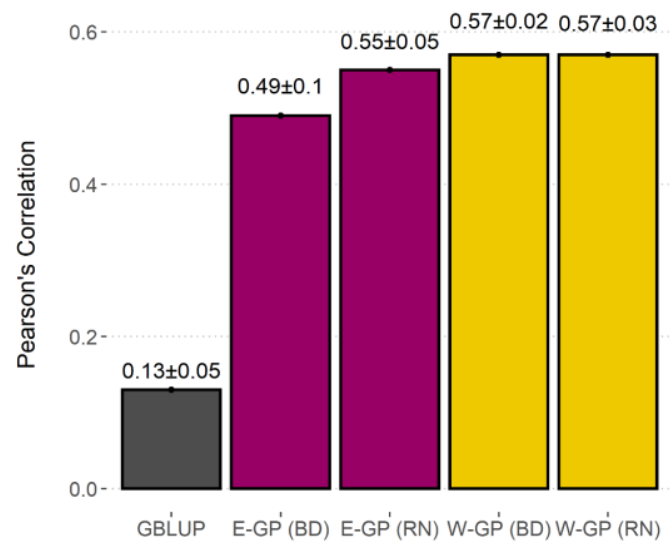

Fig. 4. Accuracy of GP models trained with super-optimized experimental networks. Predictive ability $(r)$ plus standard deviation measured by the correlation between observed and predicted values for each model in the optimized Multi-Regional Set (a); and for the N level Set (b). Barplots were colored according to the type of environmental covariable (ECs) used: none (black), envirotype descriptor (T matrix, wine), and quantitative descriptor (W matrix, 

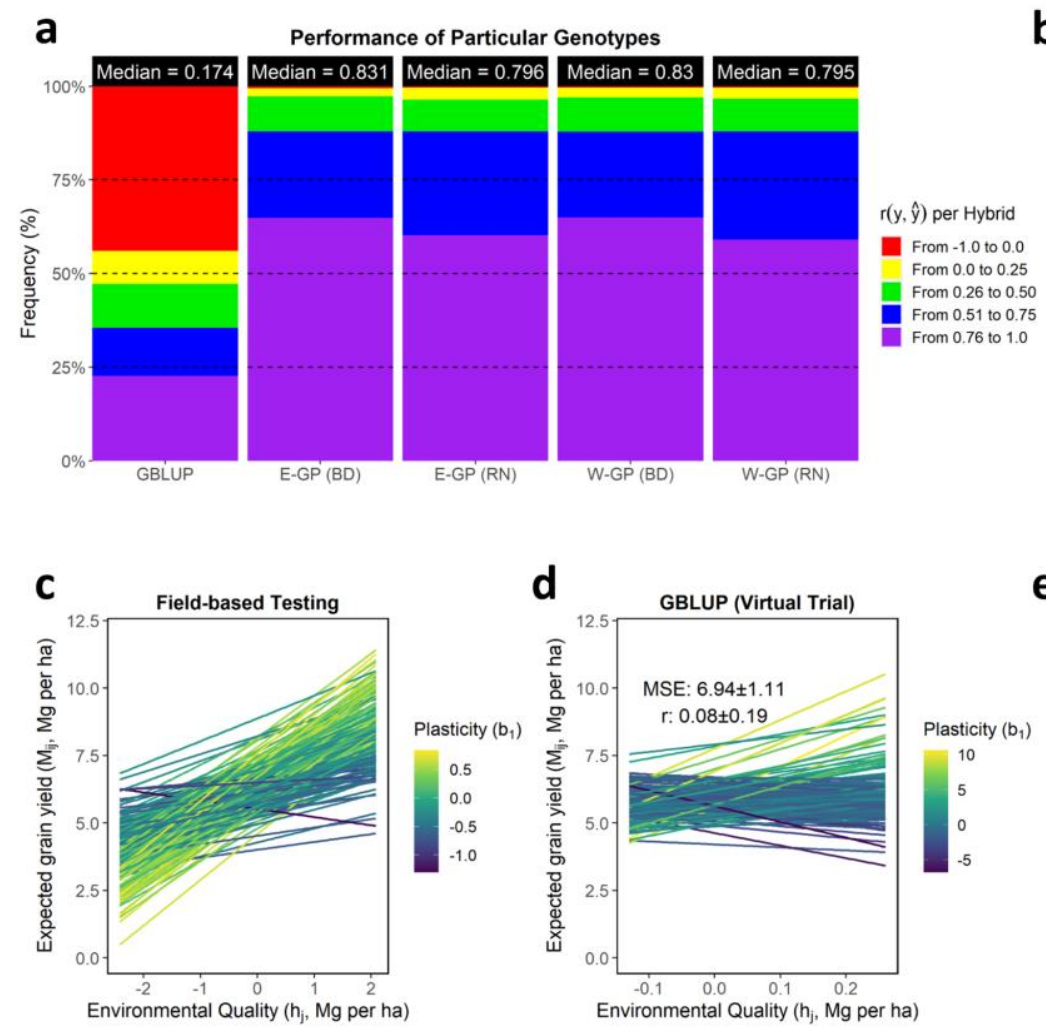

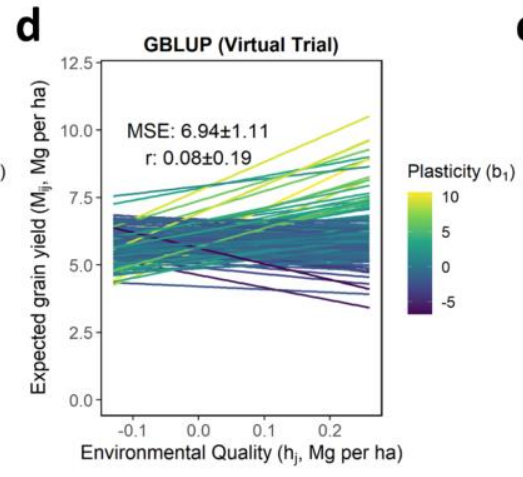

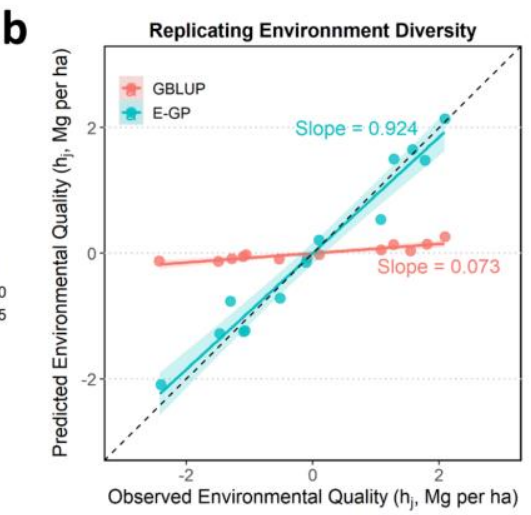

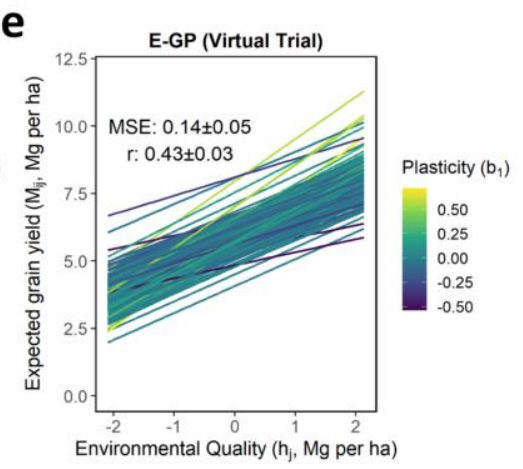

1074 Fig. 5. Accuracy of GP models in reproducing the genotype-specific plasticity. a. The panel of predictive ability $(r)$ explaining the plasticity of genotypes across environments. This statistic was estimated for each individual (hybrid) by correlating observed and predicted values across environments. Individuals with values below 0 were considered unpredictable and marked in red. b. ability of the prediction-based tools to reproduce an existing experimental network's environmental quality $\left(h_{j}\right)$. In the X-axis, we find the $h_{j}$ computed using the phenotypic records of a current experimental network. In the Y-axis, the $h_{\mathrm{j}}$ values are presented considering a virtual experimental network built up using GBLUP and E-GP (with BD) predictions. c-e. Yield plasticity panels denoting each genotype's G×E effects across the $h_{j}$ values for observed field-testing screening (c) concerning prediction-based (d-e). Only the 5\% best genotypes in each environment were used to create this plot. Each line was colored with the genotype-specific plasticity coefficient $\left(b_{1}\right)$. For the N-level set, the full-optimized set (536 hybrids over eight environments) was used. 